\title{
Seismic imaging of reservoir flow properties: Time-lapse amplitude changes
}

\author{
D. W. Vasco*, Akhil Datta-Gupta ${ }^{\dagger}$, Ron Behrens ${ }^{\ddagger}$, Pat Condon ${ }^{\S}$ and James Rickett** \\ ABSTRACT
}

Asymptotic methods provide an efficient means by which to infer reservoir flow properties, such as permeability, from time-lapse seismic data. A trajectory-based methodology, much like ray-based methods for medical and seismic imaging, is the basis for an iterative inversion of time-lapse amplitude changes. In this approach a single reservoir simulation is required for each iteration of the algorithm. A comparison between purely numerical and the trajectorybased sensitivities demonstrates their accuracy. An application to a set of synthetic amplitude changes indicates that we can recover large-scale reservoir permeability variations from time-lapse data. In an application of actual time-lapse amplitude changes from the Bay Marchand field in the Gulf of Mexico we are able to reduce the misfit by $81 \%$ in twelve iterations. The time-lapse observations indicate lower permeabilities are required in the central portion of the reservoir.

Keywords (Reservoir characterization)

${ }^{*}$ Earth Sciences Division, Berkeley Laboratory, Berkeley, CA 94720

${ }^{\dagger}$ Department of Petroleum Engineering, Texas A \& M University, College Station, TX

${ }^{\ddagger}$ ChevronTexaco Petroleum Technology Company, San Ramon, CA

${ }^{\S}$ ChevronTexaco Petroleum Technology Company, San Ramon, CA

${ }^{* *}$ ChevronTexaco Petroleum Technology Company, San Ramon, CA 


\section{INTRODUCTION}

It has been appreciated for some time that saturation and fluid pressure changes in a reservoir can lead to detectable changes in seismic attributes (Domenico 1974, Nur 1989). Correspondingly, there has been a gradual advancement in the use of time-lapse seismic observations to monitor reservoir processes. Early studies, such as the work of Greaves and Fulp (1987) on the monitoring of a fireflood, focused on detection and the estimation of basic features such as the propagation direction of the fireflood. Time-lapse seismic in now a useful tool in monitoring thermal processes (Eastwood et al. 1994, Mathisen et al. 1995, Lee et al. 1995), $\mathrm{CO}_{2}$ flooding (Lazaratos and Marion 1997, Benson and Davis 2000), gas driven production (Burkhart et al. 2000), and water driven production (Behrens et al. 2001) and more complex combinations (Johnston et al. 1998, Smith et al. 2001) including stress changes (Watts et al. 1996, Guilbot and Smith 2002). Time-lapse monitoring is maturing as a technology and guidelines for its application are available (Lumley et al. 1997). Thus, time-lapse monitoring is becoming a tool in reservoir management (Fanchi 2001). Still, the methodology is advancing and new techniques are under development which allow for discrimination between pressure and fluid saturation changes over time (Brevik 1999, Tura and Lumley 1999, Landro 2001).

To date, time-lapse seismic has chiefly served as a monitoring tool. That is, time-lapse seismic observations are used to map changes in reservoir saturation and pressure. The next logical step is to use time-lapse seismic data to characterize the reservoir, to infer reservoir permeability and porosity heterogeneity. Such time-lapse generated models of reservoir permeability aid in optimizing secondary recovery of by-passed oil and gas. The higher resolution provided by the time-lapse data can provide more accurate predictions of the performance of future production efforts. Currently, there have only been a handful of attempts at formal reservoir characterization using time-lapse observations (Landa and Horne 1997, Huang et al. 1998, He et al. 1998). By formal reservoir characterization, we mean some manner of inversion of the time-lapse data for reservoir flow properties. Timelapse reservoir characterization is hampered by computational difficulties. Typically, finding a reservoir model which is compatible with a set of saturation and pressure changes, as would be derived from the time-lapse data, requires a significant number of reservoir simulations. Because each reservoir simulation can take hours if not days of CPU time on a workstation, formal inversion can be prohibitive. For example, stochastic methods, such as the simulated annealing approach used by Huang et al. (1998), require hundreds, if not thousands of reservoir simulations.

In this paper we introduce a new trajectory-based approach for time-lapse reservoir characterization. The methodology, which is akin to ray methods used in med- ical and seismic imaging, is extremely efficient. In fact, just a single reservoir simulation is required to computed the trajectories and thus take a step in the inversion algorithm. The trajectories, which are closely related to streamlines, may be computed directly from the output of a numerical reservoir simulator. The trajectorybased approach for inverting time-lapse observations is an extension of techniques developed for the inversion of dynamic reservoir engineering data such as tracer data (Vasco and Datta-Gupta 1999), two-phase flow data (Vasco et al. 1999), and transient pressure data (Vasco et al. 2000). Here we implement the methodology for time-lapse seismic data and illustrate the approach by an application to synthetic time-lapse amplitude changes. Finally, we apply the technique to a set of time-lapse amplitude changes from the Bay Marchand field in the Gulf of Mexico.

\section{METHODOLOGY}

In this section we outline our approach for imaging reservoir flow properties using time-lapse seismic observations. First, we shall describe the method used to compute changes in elastic properties for a poroelastic medium induced by changes in fluid saturations and pressure. For this, we adopt the commonly used Gassmann's model (Gassmann 1951), simple mixing laws, and laboratory-derived relationships. The methodology for computing the seismic response of the reservoir interval and surrounding layers involves an approximation to the response of stratification, as given by Kennett (1983). We compute the seismic response of the reservoir in a piecewise fashion. That is, we compute a one-dimensional response for each column of cells in the reservoir model. The novel aspect of our approach concerns the relationship between reservoir saturation and pressure changes and reservoir flow properties. We utilize a trajectory-based technique, which is somewhat akin to seismic and electromagnetic ray methods, to relate reservoir porosities and permeabilities to saturation and pressure variations. In many cases the trajectories may be identified with streamlines, e.g. flow lines through the reservoir (King and Datta-Gupta 1998).

\section{Petrophysical Model and Seismic Amplitude Calcu- lations}

The key reservoir properties influencing its elastic response are the saturations of the various fluids in the reservoir, the pressure in the reservoir, and the porosity within the reservoir. We shall only consider the variations in water and oil saturation, denoted by $S_{w}$ and $S_{o}$, respectively. This simplifies the presentation and in our application to the Bay Marchand the influence of gas in the reservoir is small and may be neglected. Because the 
saturations sum to unity in the pore spaces we shall just consider the water saturation, which is denoted in what follows by $S$, the oil saturation is then given by $1-S$. We denote the reservoir pore pressure by $P(\mathbf{x}, t)$ and the porosity by $\phi(\mathbf{x})$.

For the frequency range and the reservoir conditions of interest Gassmann's equation (Gassmann 1951) is adequate. Gassmann's equation relates the bulk modulus of the fluid saturated rock $\left(K_{s a t}\right)$ to the moduli of the fluid mixture $\left(K_{\text {fluid }}\right)$, the moduli of the dry rock $\left(K_{d r y}\right)$, the moduli of the rock grains $\left(K_{\text {grains }}\right)$, and the porosity

$$
\begin{gathered}
K_{\text {sat }}=K_{d r y}+ \\
\frac{\left(1-K_{d r y} / K_{\text {grains }}\right)^{2}}{\phi / K_{\text {fluid }}+(1-\phi) / K_{\text {grains }}-K_{d r y} / K_{\text {grains }}^{2}} .
\end{gathered}
$$

The composite fluid bulk modulus is computed from the individual components using the simple mixing rule

$$
\frac{1}{K_{\text {fluid }}}=\frac{S}{K_{w}}+\frac{1-S}{K_{o}}
$$

where $K_{w}$ and $K_{o}$ are the bulk moduli of the water and oil respectively. The shear velocity is unaffected by changes in the composition of the pore fluid according to Gassmann's derivation. However, we should note that the dry frame moduli of both the shear velocity $\left(G_{d r y}\right)$ and the bulk sound speed $\left(K_{d r y}\right)$ are functions of the porosity and the differential (lithologic-pore) pressure $\left(P_{\text {diff }}\right)$. We assume that, as is the case at Bay Marchand (Behrens et al. 2001), the dependence of the dry moduli on porosity and effective pressure are determined from laboratory observations made on cores. The linearized functional forms used to fit the observations are

$$
\begin{aligned}
& K_{d r y}=a+b \cdot P_{d i f f} \\
& G_{d r y}=c+d \cdot P_{d i f f}
\end{aligned}
$$

where

$$
\begin{aligned}
& a=a_{0}+a_{1} \cdot P_{\text {diff }} \\
& b=b_{0}+b_{1} \cdot P_{\text {diff }} \\
& c=c_{0}+c_{1} \cdot P_{\text {diff }} \\
& d=d_{0}+d_{1} \cdot P_{\text {diff }}
\end{aligned}
$$

and the numerical values of $a_{0}, a_{1}, b_{0}, b_{1}, c_{0}, c_{1}, d_{0}$, and $d_{1}$ are the result of a nonlinear regression based upon the laboratory measurements.

The density of the saturated rock is given by the weighted average of the densities of the components

$$
\rho=\phi S \rho_{w}+\phi(1-S) \rho_{o}+(1-\phi) \rho_{\text {grains }}
$$

where $\rho_{w}, \rho_{o}$, and $\rho_{\text {grains }}$ are the respective densities for water, oil, and the component grains. The compressional $\left(V_{p}\right)$ and shear velocities $\left(V_{s}\right)$ are then computed for an isotropic elastic medium (Kennett 1983)

$$
\begin{gathered}
V_{p}=\sqrt{\frac{K_{s a t}+\frac{4}{3} G_{d r y}}{\rho}} \\
V_{s}=\sqrt{\frac{G_{d r y}}{\rho}} .
\end{gathered}
$$

Figure 1 displays the variation in compressional $\left(V_{p}\right)$ velocity as a function of saturation and differential pressure for conditions similar to those at the Bay Marchand (Behrens et al. 2001). In this figure we see that the compressional velocity is relatively insensitive to differential pressure. The compressional velocity is more strongly influenced by variations in water saturation. This will be particularly important at Bay Marchand, where there is a strong water drive and the pressure does not vary by more than $100-200$ psi. The compressional impedance, which is just the product of the compressional velocity and the density, $\left(V_{p} \cdot \rho\right)$, displays a similar behavior as the differential pressure and water saturation are varied (Figure 1).

In order to compute the seismic response of the reservoir and variations in seismic amplitudes induced by production related processes, we adopt the approach of Kennett (1983). In general, the layers in our reservoir model can be quite thin, a fraction of a meter, relative to the dominant seismic wavelengths (tens of meters). In addition, the lateral dimension of the cells in our reservoir model are of the order of tens to a hundred meters. We compute the seismic amplitudes for each vertical column of the reservoir model, approximating the response for a stack of layers. The approach involves a partial expansion of reverberation operators (Kennett 1983, p. 217) and allows for reflections at non-zero offsets, internal multiples, and tuning effects within the reservoir interval. The method is quite efficient and allows us to compute the seismic response for each column of the reservoir model in a fraction of a CPU second.

\section{Reservoir Saturation and Pressure Variations and Reservoir Flow Properties}

The relationships given above enable us to relate reservoir saturation and pressure changes to seismic amplitude changes. These relationships form the basis for most studies utilizing time-lapse seismic observations to monitor reservoir production. If, however, we wish to infer reservoir flow properties themselves, such as porosity and permeability, from time-lapse seismic observations, we must relate saturation and pressure changes to flow properties. In general, the relationship between reservoir 
flow properties and saturation changes is non-linear and involves the use of a complex numerical flow simulator. To date, this has limited the direct use of time-lapse seismic observations in reservoir characterization (Huang et al. 1998, He et al. 1998). In this sub-section we utilize a trajectory-based technique which provides an analytic relationship between reservoir flow properties and saturation changes. The approach is the basis of an efficient formalism for the inversion of reservoir production data such as water-cut observations (Vasco et al. 1999, Vasco and Datta-Gupta 2001a), as well as tracer (Vasco and Datta-Gupta 1999), and transient pressure data (Vasco et al. 2000, Vasco and Datta-Gupta 2001b).

The essential ideas underlying this approach are similar to those utilized for high frequency approximations in seismic wave propagation. There are a number of ways to motivate this technique but the main idea is the same (Anile et al. 1993). That is, the temporal and spatial variations associated with a propagating front, in this case a two-phase fluid front, are much more rapid than the variations in the background saturation distribution $\left(S_{0}\right)$. Stated another way, the jump in saturation across the two-phase front is 'fast' with respect to the changes in the background saturation. Indeed, the advancing water can form a self-sharpening front in which the water saturation jumps, almost discontinuously, from the background value to a large value. In the Appendix the idea of 'rapid' and 'slow' scales is expressed in the form of an asymptotic expansion in a scale parameter $\varepsilon$. As in elastic and electromagnetic wave propagation (Kline and Kay 1965), this approach leads to solutions which are defined along trajectories or rays (Chapman et al. 1999). As indicated by equation (A7) in the Appendix, the trajectories depend on the flow velocity field $\mathbf{U}$ which in turn is a function of the pressure distribution in the reservoir. Thus, the trajectories are similar to streamlines which are increasingly used to model reservoir flow (King and Datta-Gupta 1998).

There are several advantages associated with our trajectory-based approach. First, as in elastic wave propagation, we obtain an analytic expression for the travel time $(\sigma)$ of the two-phase front along a trajectory $\Sigma$,

$$
\sigma=\int_{\Sigma} \frac{\phi(r)}{\kappa K(r)|\nabla P|} d r
$$

where $\phi(r)$ is the porosity, $K(r)$ is the absolute permeability, $P$ is the pore pressure, and $r$ is the distance along the trajectory $\Sigma$ [see equation (A11) in the Appendix]. The variable $\kappa$ is the total mobility, a function of the relative permeabilities $k_{r n}, k_{r w}$ and the viscosities $\mu_{n}$, $\mu_{w}$

$$
\kappa=\frac{k_{r n}}{\mu_{n}}+\frac{k_{r w}}{\mu_{w}} .
$$

Thus, we have an expression for the travel time along a trajectory $\Sigma$ in terms of reservoir flow properties and the reservoir pressure distribution. Secondly, by transforming the two-phase flow problem into characteristic coordinates, coordinates oriented with respect to the trajectories, we obtain a semi-analytic expression for the saturation history at a point on the trajectory [equation (A15) in the Appendix]

$$
S(t, \sigma)=S\left(\frac{\sigma}{t}\right)
$$

where $\sigma$ is given by (8). Thirdly, the sensitivities that are required to solve the inverse problem follow from the form of the solution (9), defined along the trajectories. Specifically, in order to better fit the observations we must relate perturbations in the model parameters, the reservoir flow properties, to perturbations in the observations, the saturations within the reservoir. Because the flow properties enter (9) through the variable $\sigma$, we can consider a perturbation in this quantity. A perturbation in $\sigma$ is related to a perturbation in saturation $\delta S$, by

$$
\delta S\left(\frac{\sigma}{t}\right)=\frac{1}{t} S^{\prime}\left(\frac{\sigma}{t}\right) \delta \sigma
$$

where the prime denotes differentiation with respect to the argument $\sigma / t$. The quantity $\delta \sigma$ follows from a perturbation of the integral (8)

$$
\delta \sigma=\int_{\Sigma} \delta p(\mathbf{r}) d r
$$

where $p(r)$ is the integrand in the integral (8)

$$
p(r, t)=\frac{\phi(r)}{\kappa K(r)|\nabla P(r, t)|} .
$$

For a total mobility $(\kappa)$ which does not vary significantly, the perturbation of $p(r, t)$ is of the form

$$
\delta p(r, t)=\frac{\partial p}{\partial \phi} \delta \phi(r)+\frac{\partial p}{\partial K} \delta K(r)+\frac{\partial p}{\partial|\nabla P|} \delta|\nabla P(r, t)|
$$

The partial derivatives may be calculated directly from the analytic form for $p(r, t)$ given above [see equations (A19) in the Appendix].

\section{Sensitivity of Time-Lapse Amplitude Changes to Reservoir Flow Properties}

Here we shall combine the results of the previous two sub-sections and obtain a linear expression relating perturbations in reservoir properties to perturbations in time-lapse seismic amplitude changes. This linear relationship forms the basis for an iterative inversion. According to our petrophysical model, the seismic amplitude response of a column of cells in our reservoir model is a function of the saturation, pressure, and porosity in each cell of the column. For the $i j$-th column of the reservoir model, let us write the amplitude response as 
$A\left(\mathbf{S}_{i j}, \mathbf{P}_{i j}, \mathbf{\Phi}_{i j}\right)$ where the vectors $\mathbf{S}_{i j}, \mathbf{P}_{i j}$, and $\mathbf{\Phi}_{i j}$ denote vectors containing the saturations, pressures, and porosities for all $k$ cells in the $i j$-th column, where $i$ is the index in the east-west direction, $j$ is the index for the north-south direction, and $k$ is the index in the vertical direction. For a given location ( $i j$ value), a perturbation in the amplitude response is the sum over the perturbations in each of the $k$ cells in the column

$$
\begin{aligned}
\delta A\left(\mathbf{S}_{i j}, \mathbf{P}_{i j}, \mathbf{\Phi}_{i j}\right) & =\sum_{k} \frac{\partial A}{\partial S_{i j k}} \delta S_{i j k}+\sum_{k} \frac{\partial A}{\partial P_{i j k}} \delta P_{i j k} \\
& +\sum_{k} \frac{\partial A}{\partial \Phi_{i j k}} \delta \Phi_{i j k}
\end{aligned}
$$

For the following derivation we shall neglect the pressure terms and only concentrate on saturation and porosity changes. This will simplify the calculations and, as indicated above, for the Bay Marchand field application the sensitivity of the amplitude changes to pressure variations is much smaller than the sensitivity to changes in saturation and may be neglected. Note that this is not always the case, in many situations we must take pressure into consideration and work with the full expression (14). Neglecting the effect of pressure perturbations, the expression for the amplitude perturbation reduces to

$$
\delta A\left(\mathbf{S}_{i j}, \mathbf{\Phi}_{i j}\right)=\sum_{k} \frac{\partial A}{\partial S_{i j k}} \delta S_{i j k}+\sum_{k} \frac{\partial A}{\partial \Phi_{i j k}} \delta \Phi_{i j k} .
$$

For time-lapse amplitude data we need to consider the state of the reservoir at two distinct times $T_{0}$ and $T_{1}$. We shall denote quantities associated with each time by super-scripts. We assume that the surveys have been cross-equalized such that the amplitudes and frequency content of the seismic traces are roughly equivalent. Then, in forming the difference $\delta A^{1}\left(\mathbf{S}_{i j}, \boldsymbol{\Phi}_{i j}\right)-$ $\delta A^{0}\left(\mathbf{S}_{i j}, \mathbf{\Phi}_{i j}\right)$ the porosity term will cancel for reservoir which have not undergone compaction. Thus, the timelapse difference takes the form of a single summation over the perturbations in saturation within each of the $k$ cells of the $i j$-th column

$$
\begin{gathered}
\delta A^{1}\left(\mathbf{S}_{i j}, \mathbf{\Phi}_{i j}\right)-\delta A^{0}\left(\mathbf{S}_{i j}, \mathbf{\Phi}_{i j}\right)= \\
\sum_{k}\left(\frac{\partial A^{1}}{\partial S_{i j k}} \delta S^{1}{ }_{i j k}-\frac{\partial A^{0}}{\partial S_{i j k}} \delta S^{0}{ }_{i j k}\right) .
\end{gathered}
$$

The partial derivatives in (16) are computed by numerical differencing. That is, by computing the amplitude using the method of Kennett (1983) at a given value of saturation and then perturbing the saturation and recomputing the amplitude. Differencing these amplitudes and dividing by the saturation perturbation produces a numerical estimate of the derivative. The quantities $\delta S^{1}{ }_{i j k}$ and $\delta S^{0}{ }_{i j k}$ are given by combining equations (10) through (13). For example, $\delta S^{1}{ }_{i j k}$ is given by

$$
\delta S^{1}{ }_{i j k}=\frac{1}{T^{1}} S^{\prime}\left(T^{1}\right) \int_{\Sigma 1_{i j k}} \delta p(\mathbf{x}) d r
$$

where $\Sigma^{1}{ }_{i j k}$ denotes the trajectory from the $i j k$-th cell to a point on the initial position of the water front. Equations (16) and (17), when combined with equations (10)(13), relate perturbations in time-lapse amplitudes to perturbations in reservoir flow properties. The sensitivities are trajectory-based, the trajectory is contained in the line integral in (17), an integral over the path $\Sigma^{1}{ }_{i j k}$ and a similar line integral over the path $\Sigma^{0}{ }_{i j k}$. Note that when there is no significant variation in reservoir pressure during the time interval between the seismic surveys, the trajectories will be virtually identical.

Let us describe how we evaluate the expressions (16) and (17) in practice. We begin with a reservoir model, consisting of $n_{x} \times n_{y} \times n_{z}$ grid blocks. For example, we might have an initial distribution of porosity and permeability determined from well logs, cores, and seismic attributes. Given the initial reservoir model and well schedules, we conduct a reservoir simulation and save the saturation and pressure histories for each grid block of the model. The nature of the reservoir simulator in not important, we simply post-process the saturation and pressure histories to define the trajectories. Consider a point at the top of the reservoir where we observe changes in the amplitude of a reflection over some time interval $T^{1}-T^{0}$. The point will be located at the top of some column of cells in the reservoir model, say the $i j$-th column, containing $k$ cells in depth. For each cell we define the trajectories by moving up the pressure gradient from the cell center. We will either end up as the edge of the reservoir model, at a water injector, or at the initial position of an aquifer. If there are changes in the pressure conditions, for example more producers or injectors are added, during the time interval we will have to recompute the pressure field when appropriate. The paths thus computed define the trajectories $\Sigma^{0}{ }_{i j k}$ and $\Sigma^{1}{ }_{i j k}$ implicitly contained in (16). We use the saturation history to compute the time derivative in equation (17) and the background flow properties, pressures, and saturations to calculate the sensitivities in (13) [See equation (A19) in the Appendix]. Given the saturation distribution in the column of cells in the reservoir model at times $T^{0}$ and $T^{1}$ we use numerical differencing to compute the partial derivatives in (16). Thus, in a single reservoir simulation we have all the quantities necessary for one step of an iterative linearized inversion algorithm.

\section{NUMERICAL CALCULATIONS}

In this section we demonstrate the correctness and utility of our trajectory-based approach. First, we compare 
the trajectory-based sensitivity estimates with purely numerical results. Sensitivities, relating perturbations in model parameters to perturbations in observations, are the basis for our iterative inversion algorithm. Therefore, it is important to verify their correctness. Next, we illustrate the usefulness of the methodology by inverting a set of synthetic time-lapse amplitudes for reservoir permeability variations. The iterative inversion enables us to image large-scale permeability variations in the reservoir based upon time-lapse seismic amplitude changes.

\section{Sensitivity Computations}

Amplitude sensitivity to saturation changes. We first examine the sensitivity of time-lapse seismic amplitude changes to perturbations in water saturation within the reservoir. As stated above, we compute sensitivities on a column by column basis, treating each column as a stratified medium and computing the approximate seismic response using a partial expansion of reverberation operators (Kennett 1983). Our reservoir model is based upon a geostatistical realization intended to mimic the Bay Marchand reservoir (Behrens et al. 2001). In particular, we modeled over 14,000 days of production using a reservoir simulator. The saturation distribution within a column of cells in the reservoir model is shown in Figure 2 for three time periods (2000, 10000, and 14100 days). The corresponding compressional velocity is also presented in Figure 2. The reservoir model consists of 23 layers spanning a depth range of $2,089 \mathrm{~m}$ to $2115 \mathrm{~m}$, approximately 30 meters in thickness. The thickness of the reservoir should be compared with a quarter wavelength of the seismic data, about 17 meters. Thus, a seismic reflection off the top of the reservoir will be influenced non-uniformly by saturation changes within the reservoir interval itself.

As mentioned above, we rely on a straight-forward numerical difference to compute amplitude sensitivities to saturation variations within the column of reservoir grid blocks. Figure 3 displays the sensitivities corresponding to the saturation variations in Figure 2. Note how the sensitivity of time-lapse amplitude variations to changes in water saturation decreases dramatically at a depth of around $2104 \mathrm{~m}$, almost 15 meters into the reservoir. This is approximately one quarter of a wavelength from the top of the reservoir. The limited sensitivity with depth into the reservoir agrees with the findings of Behrens et al. (2001). They found that the best correlation coefficient was obtained when properties were averaged over the top 17 meters of the reservoir. Note also that the sensitivities depend on the water saturation within the reservoir. In particular, the sensitivity of time-lapse amplitude changes to water saturation grows with increasing water content.

In an effort to examine the numerical stability of our finite difference approximations we varied the magnitude of the perturbations used to compute the derivatives. In Figure 4 three sets of estimates are shown, corresponding to the saturation distribution at 141000 days. It is clear that for saturation perturbations from 2 percent to 10 percent, the overall behavior is the same. For example, all the curves have the rapid decay at about 2104 $m$ in depth. Though the magnitude of the perturbation changes by five times, the estimates are all within approximately 5 percent of each other. Thus, the difference estimates appear to be relatively stable.

Amplitude sensitivity to porosity and permeability changes. The trajectory-based technique is quite different from other methods for computing sensitivities to reservoir flow properties. The approach involves a 'high frequency' approximation, neglects the contribution of pressure variations to the amplitude changes, and neglects the shifting of trajectories induced by perturbations in porosity and permeability. It is important to verify that the sensitivities computed using our trajectorybased approach are accurate and are reasonable approximations to the exact sensitivities. Here we compare our trajectory-based estimates to sensitivities computed using a numerical differencing technique. The numerical results are obtained by simply perturbing the reservoir porosities and permeabilities and computing the amplitude changes observed at two particular times. The ratio of the change in the differential amplitude to the change in porosity or permeability provides a numerical estimate of the sensitivity. The differencing methodology is simple to implement and accurate but computationally intensive, requiring $N+1$ reservoir simulations where $N$ is the number of grid blocks in the reservoir model. The results of the numerical differencing do provide an important check on our trajectory-based results.

We computed sensitivities with respect to reservoir porosities and permeabilities (Figures 5 and 6). The well configuration was that of a five-spot with a central water injection well and four producing wells at the corners. The reservoir model is a homogeneous layer of 31 by 31 grid blocks. The spatial dimensions of the layer are $1 \mathrm{~km}$ by $1 \mathrm{~km}$ in area and 30 meters thick. The permeability of the layer is 150 milli-Darcies and the porosity is 10 percent. The incoming wave has a dominant frequency of $100 \mathrm{hz}$ and we treat the reflection of the compressional wave. We consider amplitude changes for seismic reflection surveys occurring 180 and 270 days after the initiation of production. Sensitivities were computed for reflections from three locations off the top of the reservoir. The left side of Figure 5 displays the results of the numeric perturbation sensitivity estimates, on the right the trajectory-based, or analytic, estimates are shown. The numeric perturbation estimates required 962 reservoir simulations while the analytic estimates result from a single simulation, almost three orders of magnitude less computation. The close agreement between the sensitivity estimates which result from these very different approaches is clear from Figure 5. The sensitivity is largest 
along a narrow zone extending from the central water injector to the point of reflection, which is denoted by a star. The nature of the sensitivities makes physical sense, we would expect the porosities along the flow path from the water injector to the reflection point to have the greatest influence on the evolution of water saturation, and hence on the amplitude changes.

The sensitivity of time-lapse amplitude changes to reservoir permeabilities is shown in Figure 6. The pattern of sensitivities in Figure 6 is similar to the porosity sensitivities, an elongated region extending from the reflection point to the water injector. However, the sign of the permeability sensitivities is opposite to that of the porosity sensitivities. This sign change agrees with the analytic expressions given by equations (A19) in the Appendix. The size of the permeability sensitivities is about three orders of magnitude smaller than the size of the porosity sensitivities. This is also in accordance with equations (A19) and the values of porosity (0.1) and permeability (150 milli-Darcies) in the layer. There are some small discrepancies between the numerical and analytic sensitivities. These are thought to be due to precision problems in computing the numeric perturbation estimates. However, they may be due to the influence of pressure variations and the shifting of the trajectories when the permeabilities are perturbed. Regardless, the differences are second order effects and there is excellent overall agreement between the two estimates.

\section{Inversion of Synthetic Time-Lapse Amplitude Changes}

We now consider a numerical illustration of the inversion of time-lapse amplitude changes. We wish to image the spatial distribution of permeability within the reservoir based upon the time-lapse data. The reference permeability for the synthetic test is shown in Figure 7 , along with the location of the producing wells (circles) and the single water injector (star). Using a reservoir simulator we modeled 1000 days of production from the layer. The initial oil saturation was 0.91 percent and the reservoir porosity was fixed at 10 percent. The distribution of water within the layer is shown after 180 and 670 days of oil production (Figure 8). The water advances along the high permeability east-central zone, extending to the south-east. The travel time of the advancing water front is shown in Figure 9. Water reaches some wells after less than 200 days, it takes over 700 days to reach other producers.

Using the method of Kennett (1983) we computed amplitudes for reflections from the top of the reservoir for two times: 180 days and 670 days. The amplitude changes during this time interval are shown in Figure 10. The largest amplitude changes occur between the location of the front at 180 days and 670 days (Figure 8). We only consider the largest fractional amplitude changes in our inversion, changes greater than a 0.015 . Such changes have a larger signal-to-noise ratio and are thus more reliable. Implementing a cutoff is similar to what is done in practice. In Figure 10 we also indicate the trajectories associated with the 105 largest amplitude changes. The trajectories extend from the reflection points to the water injector, traveling up the pressure gradient, which is also show in the figure. The trajectories, which are computed after a single reservoir simulation, are the critical elements needed to take a step in the inversion algorithm. In particular, the trajectories define the path of integration $\Sigma^{0}{ }_{i j k}$ and $\Sigma^{1}{ }_{i j k}$ in equations (16) and (17).

The 105 amplitude changes form our basic set of observations which we shall use to infer reservoir permeabilities. We adopt an iterative linearized inverse method to match the observed time-lapse amplitude changes. That is, we start with an initial reservoir model and iteratively update the permeabilities in order to better fit the data. At each step we solve a penalized least squares problem for the updates to the permeability model (Parker 1994). The inverse problem is regularized through the addition of roughness and model norm penalty terms. The sensitivities, describe above, are crucial in this iterative algorithm, for they indicate the manner in which we should modify the permeabilities in order to reduce the misfit. For each iteration of the algorithm we conduct a reservoir simulation to recompute the trajectories and redefine the pressure and saturation histories in each grid block. Our starting model is a homogeneous layer with an initial permeability of 100 milli-Darcies. The misfit reduction as a function of the number of iterations is shown in Figure 11. The misfit is reduced by about two orders of magnitude after 30 iterations. However, because the problem is non-linear, the reduction is not monotonic and some steps result in a larger misfit. That is, the model updates reduce the misfit for the linearized problem but when the model is updated and the reservoir simulator re-run the misfit actually increases. Overall, the misfit is substantially reduced after 15 iterations, equivalent to 15 reservoir simulations. The initial and final fits to the amplitude changes is shown in Figure 12. For the most part, the initial predicted amplitude changes are much lower than the actual amplitude changes. After the inversion we match all but one amplitude change.

The final reservoir permeability model is shown in Figure 13. The model contains the large-scale features of our reference model (Figure 7). In particular, there is a high permeability anomaly running to the south-east and lower permeability to the west of the injection well. In general, the resolution of heterogeneity is limited along the trajectories. That is, we cannot localize features on the trajectories. Rather, the anomalies are smeared out along the flow paths. In addition, we cannot resolve flow properties beyond the edge of the final location of the water front. In order to resolve more detailed features we require additional time-lapse surveys. Note that we can use the trajectories to efficiently compute the resolution and uncertainty associated with our estimates (Parker 
1994, Datta-Gupta et al. 2002).

\section{APPLICATION: BAY MARCHAND}

\section{The Bay Marchand Field and Time-Lapse Surveys}

The Bay Marchand is a mature field characterized by complex geologic structure. Production began in 1949 and has continued up to the present. We shall concentrate on fault block $\mathrm{A}$, one of the many stacked reservoirs at the northern flank of the field. The reservoir is a regressive marine sequence of sands and shales which pinches out against a salt dome to the south of the field. The particular interval we shall work with is the 7100' sand which is roughly 20 to 30 meters thick. The mean porosity of the sand is 30 percent and the concentration of channel sands diminishes to the west. Strong water drive from a down-dip aquifer to the north and east maintains the pressure in the reservoir and assists in the production. The 7100 ' sand is underlain by a deeper reservoir, the 7600 ' sand. Numerous wells penetrate the 7100' sand on their way to the 7600 ' sand, providing valuable log data for reservoir characterization. In all, seven wells were active at various times in the 7100' sand from 1949 until 2000 (Figure 14a). The well configuration changes over the life of the field as indicated by the water-cut history.

An initial 3D survey was conducted in 1987 as part of a program to increase production and improve the stratigraphic model of the reservoir. Subsequently, a regional 3D seismic survey extending across Bay Marchand was conducted. The geometries of the two surveys are quite different, complicating the interpretation of the timelapse changes. The first (1987) survey used seabed hydrophones which were oriented at 046 degrees, with a bin spacing of 17 meters by 17 meters. The 1987 data were only available as post-stack legacy data and could not be re-processed to enhance the time-lapse signal (Behrens et al. 2001). The subsequent 1998 Geco survey was part of a larger nonexclusive survey and was not designed for the purposes of time-lapse monitoring. The survey lines were oriented at 030 degrees with a bin spacing of 34 by 34 meters. The two surveys were re-interpolated onto a common geometry and cross-equalized in order to enhance the time-lapse effects (Behrens et al. 2001). The cross-equalization was applied to the stacked data and primarily involved static and phase shift corrections and trace equalization in a 400 to $1200 \mathrm{mS}$ time window.

Some idea of the repeatability or similarity of the surveys is apparent in Figure 14b where we superimpose fourteen traces from the two surveys. Overall, traces from the two surveys are remarkable similar, suggesting that the repeatability is quite good overall. A more quantitative study was undertaken by Behrens et al. (2001) who examined the signal to noise ratio, the root-meansquare differences, and the correlation between the surveys. Amplitudes were extracted for a reflection from the top of the 7100' sand. The horizon is denoted by the stars in Figure 14b. The amplitude change is defined as the amplitude of the 1998 survey subtracted from the amplitude of the 1987 survey. The peak amplitude change in a $20 \mathrm{mS}$ window was taken as the amplitude difference. We averaged the amplitude changes within the lateral boundaries of our reservoir model to further enhance the signal. The results are shown in Figure 15 for cells which do not lie within the aquifer. We observe significant changes near the production wells in this figures. Fractional amplitude changes of over 5 percent are observed over the reservoir proper.

\section{The Reservoir Model at Bay Marchand}

As discussed by Behrens et al. (2001), a reservoir model was carefully constructed for the 7100 ' sand at Bay Marchand. Because of the deeper producing 7600' sand, there were some fifty logged wells penetrating the 7100 'sand yet not producing from it. Detailed facies logs and maps were used to simulate petrophysical facies and generate geostatistical reservoir models. The reservoir model consisted of twenty-three layers and predictions based upon it could generally match the cumulative production data. For our preliminary study we averaged the reservoir properties of the twenty-three layers to produce a single layer reservoir model. The porosity and saturations in each layer were linearly averaged together while the logarithm of the permeabilities were averaged. The resulting permeabilities and initial water saturations are shown in Figures 16a and 16b, respectively, along with the locations of the active producing wells (circles) and an observation well for which a time-lapse pulsed neutron log was available (star). As noted above, the quality of the reservoir diminishes to the west and this is notable in Figure $16 \mathrm{a}$ as a sharp decrease in permeability. In addition, because the reservoir pinches out to the south as the salt dome is approached, the permeability at the southern margin the of model is quite low. The initial location of the aquifer is indicated by the high water saturation in Figure 16b.

\section{Inversion of the Time-Lapse Data}

Using the methodology outlined above, we inverted the amplitude changes between the two seismic surveys. Due to the nature of the surveys, the initial survey consists of legacy data gathered after the onset of production, the amplitude changes are rather noisy. Therefore, we used the largest amplitude changes in the inversion. In particular, twenty-one of the largest amplitude changes comprised our basic data set. Starting from the initial permeability model shown in Figure 16a we iteratively updated the permeabilities in order to better fit the amplitude changes. The misfit reduction as a function of the 
number of iterations is shown in Figure 17. The squared misfit is reduced by $81 \%$ in twelve iterations. The final fit to the amplitude changes is indicated in Figure 18. Initially, the amplitude changes are systematically under-predicted. The final fit still contains considerable scatter. One reason for this could be that we are fitting the amplitude changes using a single layer model. We expect the fit to improve when additional layers are added to the reservoir model. Also, because the two surveys were not designed for time-lapse monitoring, the data are rather noisy in general. The final model, shown in Figure 19a, contains generally lower permeabilities in the central region of the reservoir. The lower permeabilities are required to slow down the arrival of the water in order to produce the largest changes within the time interval between the two seismic surveys.

The predicted amplitude changes, based upon the permeability model from 19a, are show in Figure 19b. Many of the general features of the observed time-lapse amplitude changes (Figure 15) are reproduced by the updated reservoir. In particular, the model significant amplitude changes are found around the observation well which is indicated by the star. Furthermore, the largest amplitude changes form an arcuate pattern extending from the observation well to the westernmost producing wells. The detailed amplitude variation is not reproduced by the updated reservoir model. For example, the amplitude changes to the north of the acuate amplitude changes are not found in the predicted amplitude changes. But, as noted above, because the legacy data could not be reprocessed for time-lapse and the second survey was not designed for time-lapse monitoring, the observed amplitude variations are rather noisy and thus, detailed interpretation may not be warranted. Also, we have adopted a simple one-layer reservoir model in this initial attempt to match the time-lapse measurements. In spite of these limitations, the time-lapse amplitude data indicate that permeabilities in the central portion of the reservoir are too large to match the observations.

\section{CONCLUSIONS}

An asymptotic approach to the modeling of the propagation of a two-phase front provides an efficient formalism with which to invert time-lapse seismic amplitude changes. Specifically, the formulation results in a trajectory-based method for using time-lapse amplitude changes to image reservoir flow properties, such as permeability. Synthetic testing and comparisons with numerical computations indicate that the inversion scheme is both efficient and accurate. We find that time-lapse seismic amplitude changes may be used to infer the largescale permeability variations in a reservoir. Our application to actual time-lapse data from the Bay Marchand field indicates that the method is robust in the presence of noise.
This work can be extended in several respects. In the future, we would like to integrate both reservoir production observations, such as water-cut and well pressure data, with seismic time-lapse measurements. It would also be interesting to incorporate pressure estimates derive from time-lapse seismic AVO (Landro 2001). We would like to extend the trajectory-based methodology to more general settings, for example, when capillary effects are present. Finally, we want to apply the methodology to additional data sets, including data from surveys designed specifically for time-lapse monitoring.

\section{ACKNOWLEDGMENTS}

This work was supported by the Assistant Secretary for Fossil Energy, Office of Oil Gas and Shale Technologies, of the U.S. Department of Energy under contract DEAC03-76SF00098. All computations were carried out at the Center for Computational Seismology. 


\section{REFERENCES}

Aki, K. and Richards, P. G., 1980, Quantitative Seismology, W. H. Freeman and Company, San Francisco.

Anile, A. M., J. K. Hunter, P. Pantano, and G. Russo, 1993, Ray Methods for Nonlinear Waves in Fluids and Plasmas, Longman Scientific and Technical, Essex.

Bedrikovetsky, P., 1993, Mathematical Theory of Oil and Gas Recovery, Kluwer Academic Publishing, Amsterdam.

Behrens, R., Condon, P., Haworth, W., Bergeron M., Wang, Z., and Ecker, C., 2001, 4D seismic monitoring of water influx at Bay Marchand: The practical use of 4D in an imperfect world: paper SPE 71329 presented at the 2001 SPE Annual Technical Conference and Exhibition, New Orleans, Louisiana, September 30-October 3 .

Benson, R. D., and Davis, T. L., 2000, Time-lapse seismic monitoring and dynamic reservoir characterization, Central Vacuum Unit, Lea County, New Mexico: SPE Res. Eval. and Eng., 3, 88-97.

Brevik, I., 1999, Rock model based inversion of saturation and pressure changes from time lapse seismic data: 69th Ann. Internat. Mtg. Soc. Expl Geophys., Expanded Abstracts, 1044-1047.

Burkhart, T., Hoover, A. R., and Flemings, P. B., 2000, Time-lapse (4D) seismic monitoring of primary production of turbidite reservoirs as South Timbalier Block 295, offshore Louisiana, Gulf of Mexico: Geophysics, 65, 351-367.

Buckley, S. E., and Leverett, M. C., 1942, Mechanism of fluid displacement in sands: Trans. Am. Inst. Mech. Engng., 146, 107-116.

Chapman, S. J., Lawry, J. M. H., and Ockendon, J. R., 1999, Ray theory for high-peclet-number convectiondiffusion: SIAM J. Appl. Math., 60, 121-135.

Chorin, A. J., and Marsden, J. E., 1990, A Mathematical Introduction to Fluid Mechanics, Springer-Verlag, Berlin.

Courant, R., and D. Hilbert, 1962, Methods of Mathematical Physics, Interscience, New York.

Datta-Gupta, A., and King, M. J., 1995, A semianalytic approach to tracer flow modeling in heterogeneous permeable media: Advan. Water Resour., 18, 9-24.

Datta-Gupta, A., Yoon, S., Vasco, D. W., and Pope, G. A., 2002. Inverse modeling of patitioning tracer tests: A streamline approach, Water Resour. Res., 38, (6), 10.1029 .
Domenico, S. N., 1974, Effect of water saturation on seismic reflectivity of sand reservoirs encased in shale: Geophysics, 39, 759-769.

Eastwood, J., Lebel, J. P., Dilay, A., and Blakeslee, S., 1994, Seismic monitoring of steam-based recovery of bitumen: The Leading Edge, 4, 242-251.

Fanchi, J. R., 2001, Time-lapse seismic monitoring in reservoir management: The Leading Edge, 20, 11401147.

Gassmann, F., 1951, Elastic waves through a packing of spheres: Geophysics, 16, 673-685.

Greaves, R. J., and Fulp, T., 1987, Three dimensional seismic monitoring of an enhanced oil recovery process: Geophysics, 52, 1175-1187.

Guilbot, J. and Brackin, S., 2002, 4-D constrained depth conversion for reservoir compaction estimation: Application to Ekofisk field: The Leading Edge, 21, 302-308.

He, W., Guerin, G., Anderson, R. N., and Mello, U. T., 1998, Time-dependent reservoir characterization of the LF sand in the South Eugene Island 330 Field, Gulf of Mexico: The Leading Edge, 17, 1434-1438.

Huang, X., Meister, L., and Workman, R., 1998, Improving production history matching using time-lapse seismic data: The Leading Edge, 17, 1430-1433.

Jeffrey, A., 1976, Quasilinear Hyperbolic Systems and Waves Pitman Publishing, London.

Johnston, D. H., McKenny, R. S., Verbeek, J. and Almond, J., 1998, Time-lapse seismic analysis of Fulmar Field: The Leading Edge, 17, 1420-1428.

Kennett, B. L. N., 1983, Seismic Wave Propagation in Stratified Media, Cambridge University Press.

King, M. J., and Datta-Gupta, A., 1998, Streamline simulation: A current perspective: In Situ, 22, 91-140.

Kline, M., and Kay, I. W., 1965, Electromagnetic Theory and Geometrical Optics, John Wiley and Sons.

Landa, J. L., and Horne, R. N., 1997, A procedure to integrate well test data, reservoir performance history, and 4-D seismic information into a reservoir description: paper SPE 38653 presented at the 1997 SPE Annual Technical Conference and Exhibition, San Antonio, Texas, October 5-8.

Landro, M., 2001, Discrimination between pressure and fluid saturation changes from time-lapse seismic data: Geophysics, 66, 836-844.

Lazaratos, S. K., and Marion, B. P., 1997, Crosswell seismic imaging of reservoir changes caused by $\mathrm{CO}_{2}$ injection: The Leading Edge, 16, 1300-1307. 
Lee, D. S., Stevenson, V. M., Johnston, P. F., and Mullen, C. E., 1995 Time-lapse crosswell seismic tomography to characterize flow structure in the reservoir during thermal stimulation: Geophysics, 60, 660666.

Lumley, D. E., Behrens, R. A., and Wang, Z., 1997, Assessing the technical risk of a $4 \mathrm{D}$ seismic project: The Leading Edge, 16, 1287-1294.

Mathisen, M. E., Vasiliou, A. A., Cunningham, P., Shaw, J., Justice, J. H., and Guinzy, N. J., 1995, Time-lapse crosswell seismic tomogram interpretation: Implications for heavy oil reservoir characterization, thermal recovery process monitoring, and tomographic imaging technology: Geophysics, 60, 631-650.

Nur, A., 1989, Four-dimensional seismology and (true) direct detection of hydrocarbon: The petrophysical basis: The Leading Edge, 8, 30-36.

Parker, R. L., 1994, Geophysical Inverse Theory Princeton University, Princeton, N.J.

Peaceman, D. W., 1977, Fundamentals of Numerical Reservoir Simulation, Elsevier Scientific Publishing.

Smith, P, Berg, J. I., Eidsvig, S., Magnus, I., Verhelst, F., and Helgesen, J., 2001, 4-D seismic in a complex fluvial reservoir: The Snorre feasibility study: The Leading Edge, 20, 270-276.

Tura, A., and Lumley, D. E., 1999, Estimating pressure and saturation changes from time-lapse AVO data: 61st Ann. Conf. Eur. Assoc. Geosci. Eng., Extended Abstracts, 1-38.

Vasco, D. W., and Datta-Gupta, A., 1999, Asymptotic solutions for solute transport: A formalism for tracer tomography: Water Resour. Res., 35, 1-16.

Vasco, D. W., and Datta-Gupta, A., 2001a. Asymptotics, saturation fronts, and high resolution reservoir characterization: Trans. Porous Media, 42, 315-350.

Vasco, D. W., and Datta-Gupta, A., 2001b. Asymptotics, streamlines, and reservoir modeling: A pathway to production tomography: The Leading Edge, 20, 11641171.

Vasco, D. W., Yoon, S. and Datta-Gupta, A., 1999. Integrating dynamic data into high-resolution reservoir models using streamline-based analytic sensitivity coefficients: SPE Jour., 4, 389-399.

Vasco, D. W., Karasaki, K., and Keers, H., 2000. Estimation of reservoir properties using transient pressure data: An asymptotic approach: Water Resour. Res., 36, 3447-3465.
Watts, G. F. T., Jizba, D., Gawith, D. E., and Gutteridge, P., 1996, Reservoir monitoring of the Magnus field through 4D time-lapse seismic analysis: Petroleum Geoscience, 2, 361-372.

Whitham, G. B., 1974, Linear and Nonlinear Waves, John Wiley and Sons, New York. 


\section{APPENDIX: ASYMPTOTIC SOLUTION FOR TWO-PHASE FLOW}

This Appendix outlines the derivation of an asymptotic solution for the propagation of a two-phase fluid front. In particular, we derive an analytic relationship between reservoir flow properties and the arrival time and amplitude variation of a propagating two-phase fluid front. This asymptotic solution is the basis for an analytic relationship between time-lapse seismic amplitude changes and reservoir flow properties.

\section{Governing Equations for Two-Phase Flow}

We begin with the pair of partial differential equations describing the flow of an wetting (water) phase and a non-wetting (oil) phase (Peaceman, 1977)

$$
\begin{gathered}
\nabla \cdot\left[\frac{\rho_{w} K(\mathbf{x}) k_{r w}}{\mu_{w}} \nabla\left(P_{w}(\mathbf{x}, t)-\rho_{w} g z\right)\right]=\phi(\mathbf{x}) \frac{\partial\left(\rho_{w} S_{w}\right)}{\partial t} \\
\nabla \cdot\left[\frac{\rho_{o} K(\mathbf{x}) k_{r o}}{\mu_{o}} \nabla\left(P_{o}(\mathbf{x}, t)-\rho_{o} g z\right)\right]=\phi(\mathbf{x}) \frac{\partial\left(\rho_{o} S_{o}\right)}{\partial t}
\end{gathered}
$$

where the variables $S_{w}$ and $S_{o}$ denote the saturations of the water and oil phases, respectively. The relative permeabilities of the water and oil phases are represented by $k_{r w}$ and $k_{r o}$ while the permeability is given by $K(\mathbf{x})$. The densities of the water and oil are $\rho_{w}$ and $\rho_{o}, g$ denotes the gravitational constant, and the spatially varying porosity is $\phi(\mathbf{x})$. The pressure associated with the wetting phase is $P_{w}(\mathbf{x}, t)$ and $P_{o}(\mathbf{x}, t)$ is the pressure associated with the oil phase, The viscosities for the water and oil phases are $\mu_{w}$ and $\mu_{o}$. The two equations are coupled because the saturations are constrained to sum to unity

$$
S_{w}+S_{o}=1
$$

If capillary forces are small relative to other factors, we may derive a single equation describing the evolution of the saturation of the wetting phase, which shall be denoted by $S(\mathbf{x}, t)$ (Peaceman 1977, Bedrikovetsky 1993),

$$
\phi(\mathbf{x}) \frac{\partial S}{\partial t}+\mathbf{U} \cdot \nabla S+C(S)=0
$$

where $\mathbf{U}$ is the flow velocity and $C(S)$ is a term related to gravity,

$$
C(S)=g \lambda_{w} \frac{\left(\rho_{w}-\rho_{o}\right)}{\lambda_{o}+\lambda_{w}} \frac{k_{r_{o}}(S)}{\mu_{o}} \frac{\partial K(\mathbf{x})}{\partial z} .
$$

The spatial variations in the relative permeability parameters, $k_{r_{o}}$ and $k_{r w}$, are assumed to occur due to variations in saturation.

\section{Asymptotic Solutions for Two-Phase Flow}

The motivation for our asymptotic solution is based upon a variation in scale (Whitham 1974, Jeffrey 1976, Anile et al. 1993). That is, we assume that the initial saturation distribution is a relatively slowly varying function of space and time when compared to the jump in saturation across the two-phase front. In effect, there is a scale, denoted by $L$, describing the variation in background saturation in time and space and a scale $\Lambda$ describing the spatial and temporal variation in saturation across the propagating two-phase front. We are assuming that $L \gg \Lambda$ holds in the domain of interest. If we denote $\Lambda / L$ by $\epsilon$, the condition is $0<\epsilon \ll 1$.

An asymptotic expansion is the representation of the solution as a formal series in powers of the parameter $\epsilon$ (Anile et al., 1993)

$$
S(\mathbf{x}, t)=S_{0}(\mathbf{x}, t)+\sum_{n=1}^{\infty} \epsilon^{n} S_{n}(\mathbf{x}, t, \omega)
$$

where $S_{0}(\mathbf{x}, t)$ represents the background variation in saturation and $\omega$ is the frequency of the wave. The frequency $\omega$ is assumed to have the form

$$
\omega=\frac{\sigma(\mathbf{x}, t)}{\epsilon}
$$

(Anile et al., 1993) where $\sigma(\mathbf{x}, t)$ is the phase variation of the wave, a function describing the geometric configuration of the propagating multi-phase front in space and time. Note that the methodology is similar to asymptotic methods in electromagnetic (Kline and Kay 1965) and seismic wave propagation (Aki and Richards 1980).

In constructing our asymptotic representation we substitute the expansion (A2) of $S(\mathbf{x}, t)$ into the various terms of equation (A1). For example, consider the components of the vector $\mathbf{U}(S)$ which may be represented as a power series in $S$. The expansion is given by

$$
\mathbf{U}(S, \mathbf{x}, t)=\mathbf{U}\left(S_{0}, \mathbf{x}, t\right)+\epsilon \frac{\partial \mathbf{U}}{\partial S} S_{1}+O\left(\epsilon^{2}\right)
$$

where $O\left(\epsilon^{2}\right)$ denotes terms of order $\epsilon^{2}$ and higher. Substituting the expansions into equation (A1) produces an equation containing an infinite sequence of terms. Each term in the sequence contains $\epsilon$ to some power as a factor.

Arrival time of the two-phase front. As shown in Vasco and Datta-Gupta (2001a), neglecting terms containing $\epsilon$ of order one or greater produces the equation

$$
\phi(\mathbf{x}) \frac{\partial \sigma}{\partial t}+\mathbf{U}\left(S_{0}, \mathbf{x}, t\right) \cdot \nabla \sigma=0 .
$$

The quantity $\sigma(\mathbf{x}, t)$ governs the propagation or kinematics of the multi-phase front. Note that the velocity vector $\mathbf{U}$ only depends on the background saturation.

Using the implicit function theorem we may write (A5) in the form $\varphi(\mathbf{X}, T)=T-\sigma(\mathbf{X})$ where $\sigma$ only depends 
on position. Then (A5) reduces to

$$
\nabla \sigma \cdot \mathbf{U}\left(S_{0}, \mathbf{x}, t\right)=\phi(\mathbf{x})
$$

a first-order linear partial differential equation, governing the distribution of $\sigma$. We may solve this equation directly, using the method of characteristics (Courant and Hilbert, 1962, p. 70). In the method of characteristics solutions are developed along particular trajectories, the characteristic curves, which are denote by $\mathbf{X}(l)$, where $l$ is a parameter signifying position along the curve. The equations for the characteristic curves are a set of four ordinary differential equations

$$
\begin{gathered}
\frac{d \mathbf{X}}{d l}=\mathbf{U}\left(S_{0}, \mathbf{x}, t\right) \\
\frac{d \sigma}{d l}=\phi(\mathbf{x})
\end{gathered}
$$

(Courant and Hilbert, 1962, p. 70). For a coordinate system with one axis oriented along $\mathbf{U}$ we can write (A7) as

$$
\frac{d r}{d l}=U
$$

where $U=|\mathbf{U}|$ and $r$ denotes the distance along the axis aligned with $\mathbf{U}$. Combining equations (A8) and (A9), we may express $\sigma$ as the integral

$$
\sigma=\int_{\Sigma} \frac{\phi(r)}{U} d r
$$

where $\Sigma$ is the trajectory from an initial point on the water front to the final point. Note that this expression for phase is similar to that in Vasco and Datta-Gupta (2001a) if we incorporate generalized Darcy's law $\mathbf{U}=$ $\kappa K \nabla P$, where $\kappa$ is the total mobility

$$
\kappa=\frac{k_{r o}}{\mu_{r o}}+\frac{k_{r w}}{\mu_{r w}} .
$$

Equation (A10) then becomes

$$
\sigma=\int_{\Sigma} \frac{\phi(r)}{\kappa K|\nabla P|} d r
$$

or

$$
\sigma=\int_{\Sigma} p(r, t) d r
$$

if we write the integral in terms of

$$
p(r, t)=\frac{\phi(r)}{\kappa K(r)|\nabla P(r, t)|}
$$

which we call the front slowness. Note that the front slowness depends on time through the time dependence of the pressure gradient.
Amplitude of the two-phase front. We may write the equation governing two-phase flow (A1) in terms of the characteristic coordinates, e.g. a coordinate system defined by the characteristic curves associated with equations (A7) and (A8) (Vasco et al. 2001a). The result is a a first-order, quasi-linear, hyperbolic equation for $S(t, \sigma)$ of the form (King and Datta-Gupta 1998)

$$
\frac{\partial F(S)}{\partial \sigma}+\frac{\partial S}{\partial t}=0
$$

where $F(S)$ is the fractional flow function

$$
F(S)=\frac{m_{w}}{m_{o}+m_{w}}
$$

for $m_{w}=k_{r w} / \mu_{r w}$ and $m_{o}=k_{r_{o}} / \mu_{r_{o}}$. Equation (A14) is invariant with respect to coordinate scalings of the type:

$$
t^{\prime}=\varepsilon t, \quad \sigma^{\prime}=\varepsilon \sigma, \quad \varepsilon>0
$$

which requires the solution to take the general form (set $\varepsilon=1 / t)$

$$
S(t, \sigma)=S\left(\frac{\sigma}{t}\right)
$$

if it is to be unique (Chorin and Marsden, 1990; Bedrikovetsky, 1993). A specific form for $S(t, \sigma)$ was derived by Buckley and Leverett (1942).

Amplitude Sensitivities: Analytic Expressions.From the general form of the solution for saturation (A15) we may compute analytic expressions for saturation amplitude sensitivities to changes in reservoir properties (Vasco et al. 1999). That is, we wish to determine how perturbations in reservoir properties, such as porosity and permeability, map into perturbations in the saturation at a point in the reservoir. The important point to note is that the reservoir properties enter the expression (A15) through the variable $\sigma$. As noted in Vasco and Datta-Gupta (2001a), a perturbation in $\sigma$ is related to a perturbation in saturation $\delta S$, by

$$
\delta S\left(\frac{\sigma}{t}\right)=\frac{1}{t} S^{\prime}\left(\frac{\sigma}{t}\right) \delta \sigma
$$

where $\sigma$ is given by the integral (A11). The quantity $\delta \sigma$ follows from a perturbation of the integral (A11)

$$
\delta \sigma=\int_{\Sigma} \delta p(r) d r
$$

where

$$
\delta p(r)=\frac{\partial p}{\partial \phi} \delta \phi(r)+\frac{\partial p}{\partial K} \delta K(r)+\frac{\partial p}{\partial|\nabla P|} \delta|\nabla P(r)|
$$

The partial derivatives may be calculated directly from equation (A13)

$$
\frac{\partial p}{\partial \phi}=\frac{p(r)}{\phi(r)}
$$




$$
\begin{gathered}
\frac{\partial p}{\partial K}=-\frac{p(r)}{K(r)} \\
\frac{\partial p}{\partial|\nabla P|}=-\frac{p(r)}{|\nabla P(r)|} .
\end{gathered}
$$

If the trajectories are not significantly perturbed by the passage of the saturation front then the perturbed trajectory $\Sigma$ may be replaced by the unperturbed trajectory $\Sigma_{0}$. This approximation is thought to be a good one for waterflood fronts (Datta-Gupta and King, 1995), and appropriate for the oil field application considered.

\section{FIGURE CAPTIONS}

Figure 1. (Left) Variation in compressional wave velocity as a function of water saturation and differential pressure. (Right) Variation in compressional wave impedance as a function of water saturation and differential pressure.

Figure 2. (Left) Saturation distribution in a column of our reservoir model for three different times: 2000, 10000 , and 14100 days. (Right) Compressional wave velocity for the corresponding column of reservoir model and three time intervals.

Figure 3. Sensitivity of time-lapse amplitude differences to saturation changes in a column of the reservoir model. The sensitivities are computed with respect to three background saturation distributions. The column and times correspond to those in Figure 2.

Figure 4. Amplitude sensitivities for saturations in a column of the reservoir model. The background saturation distribution is that of 14100 days in Figure 2. The sensitivities are computed using three different saturation perturbations: $0.02,0.05$, and 0.10 .

Figure 5. Numeric (left) and trajectory-based analytic (right) amplitude sensitivity to changes in reservoir porosity. The amplitudes correspond to reflections from three different points, denoted by the stars.

Figure 6. Numeric (left) and trajectory-based analytic (right) amplitude sensitivity to changes in reservoir permeability. The amplitudes correspond to reflections from three different points, denoted by the stars.

Figure 7. Reference permeability field used to compute synthetic time-lapse amplitude changes.

Figure 8. Water saturation distributions after 180 and 670 days of oil production from the reservoir.

Figure 9. The travel time of the water phase as it flows from the injection well (star) to the producing wells (circles).

Figure 10. (Upper) Pressure in the reservoir after 180 days of production. (Lower) Amplitude changes between 180 and 670 days of production. The curves in this figure are the trajectories used for the inversion of the amplitude values. They travel up the pressure gradient from the reflection point to the injection well.

Figure 11. Squared misfit as a function of the number of iterations of the inversion algorithm.

Figure 12. Observed time-lapse amplitude changes plotted against the calculated amplitude changes. The initial fits (open circles) are based on the initial homogeneous reservoir model. The final fits (filled squares) are computed using the final iteration of the inversion algorithm.

Figure 13. Final permeability model which results from an inversion of the synthetic time-lapse amplitude changes.

Figure 14a. Water-cut from the seven wells producing from the 7100 ' sand at Bay Marchand. 
Figure 14b. Selected traces from a base survey (solid) shot in 1987 at Bay Marchand. Corresponding traces from a regional survey (dashed) shot in 1998 for a region encompassing Bay Marchand.

Figure 15. Peak amplitude changes between 1987 and 1998 for the 7100' sand at the Bay Marchand.

Figure 16. (A) Initial permeability model for the 7100 ' sand at the Bay Marchand. (B) Initial water saturation prior to the start of production at the Bay Marchand.

Figure 17. Squared misfit as a function of the number of iterations of the inversion algorithm.

Figure 18. Initial (open circles) and final (solid squares) fits to the Bay Marchand time-lapse amplitude changes.

Figure 19. (A) Final permeability model resulting from an inversion of the time-lapse amplitude changes. (B) Fractional amplitude changes predicted by the final inversion results. 

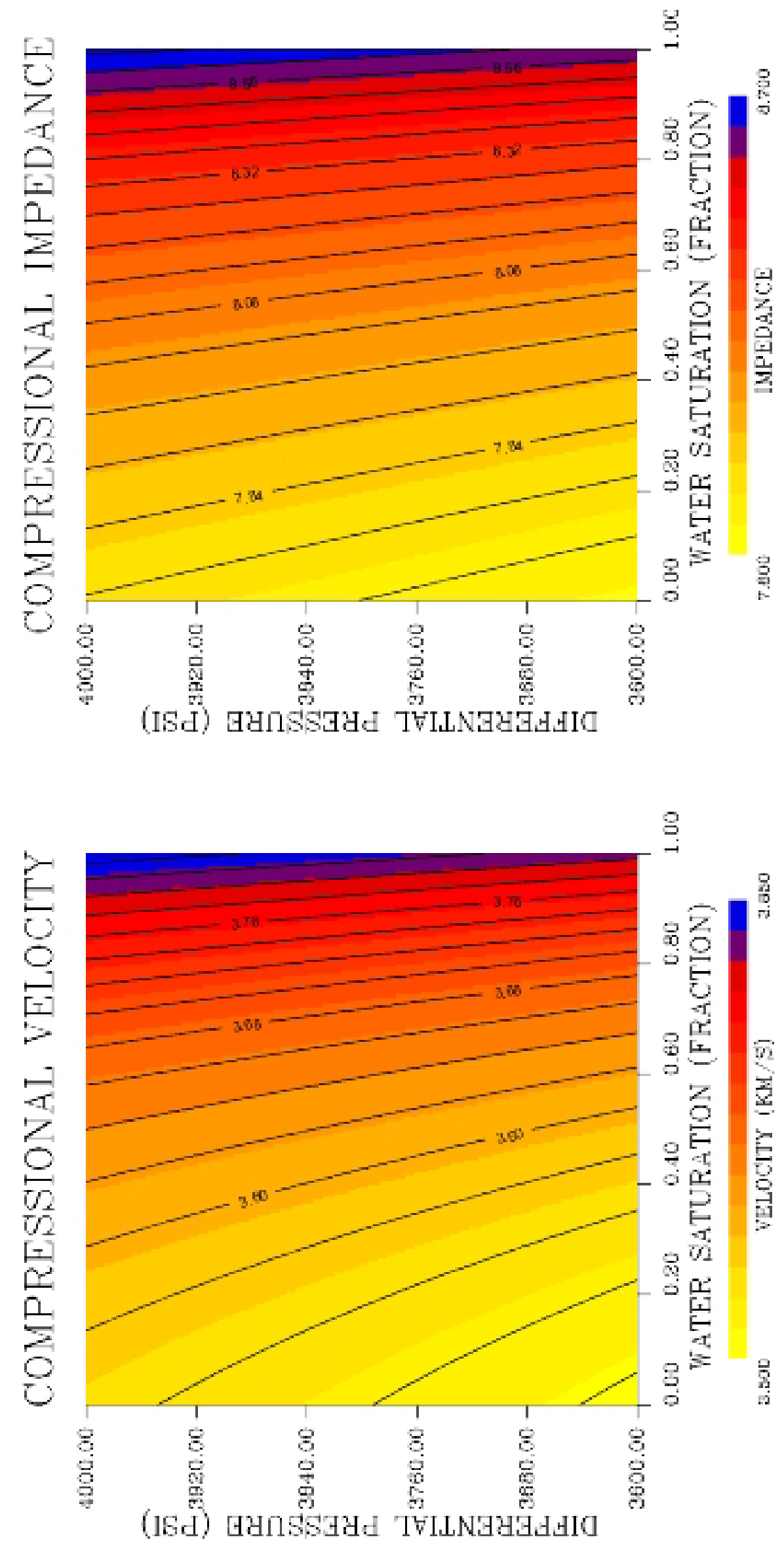

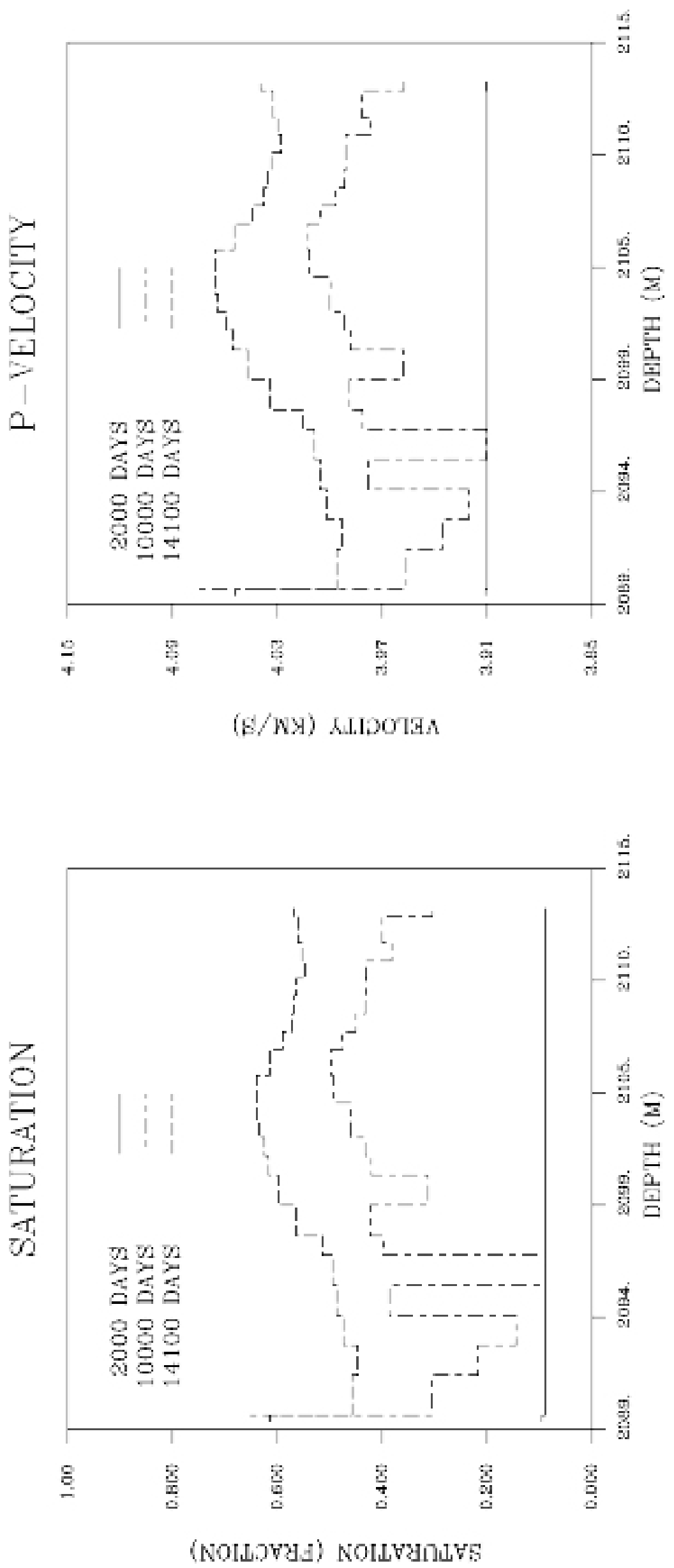


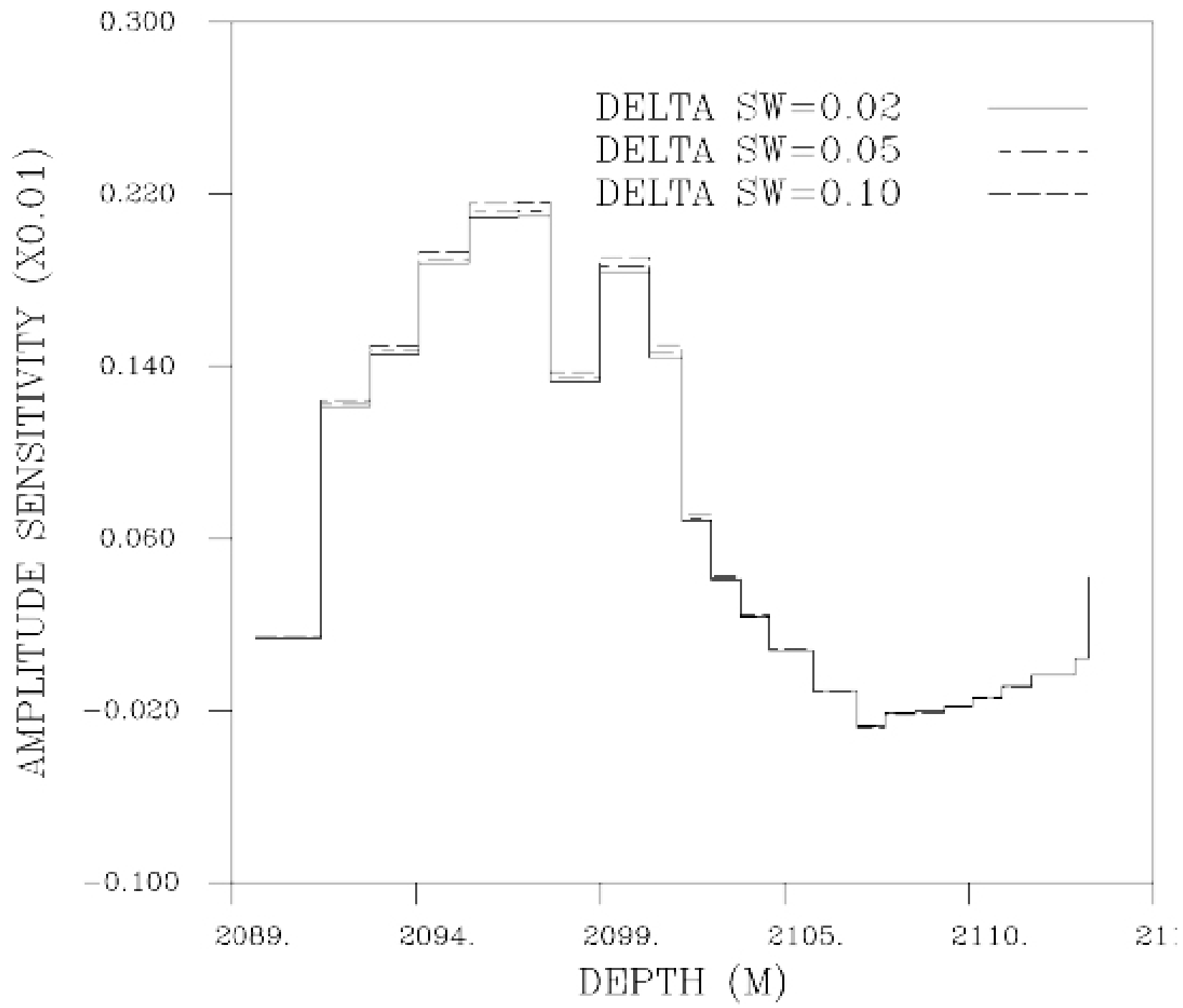




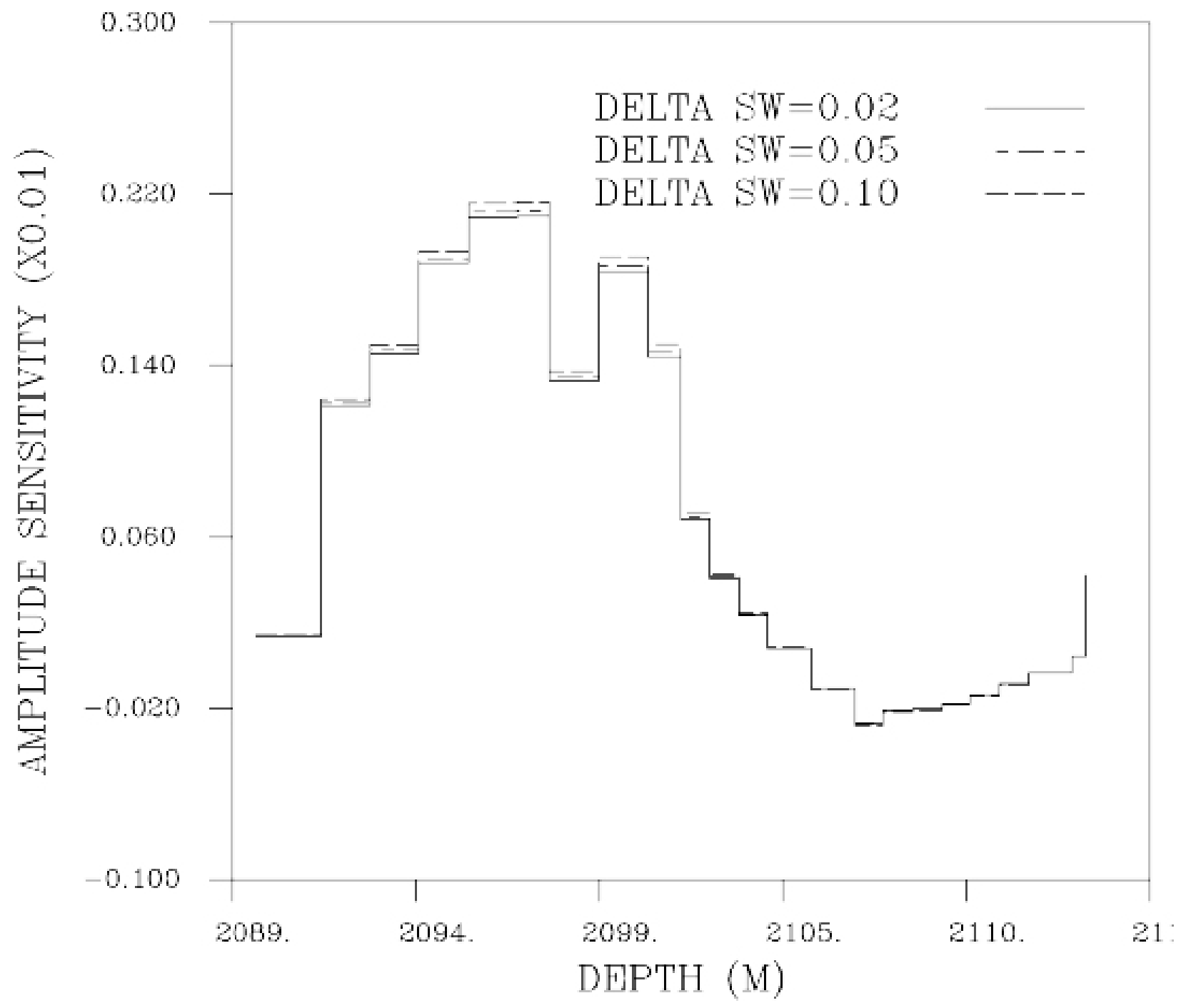




\section{Porosity Sensitivities}

\section{Numeric}
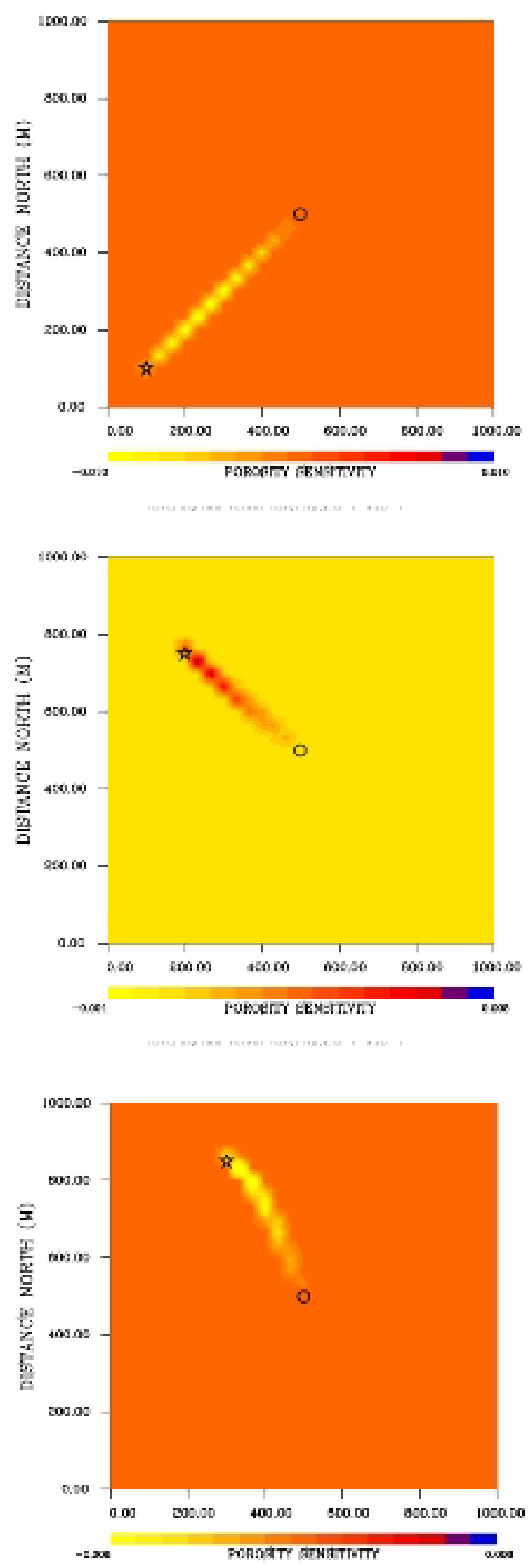

Analytic
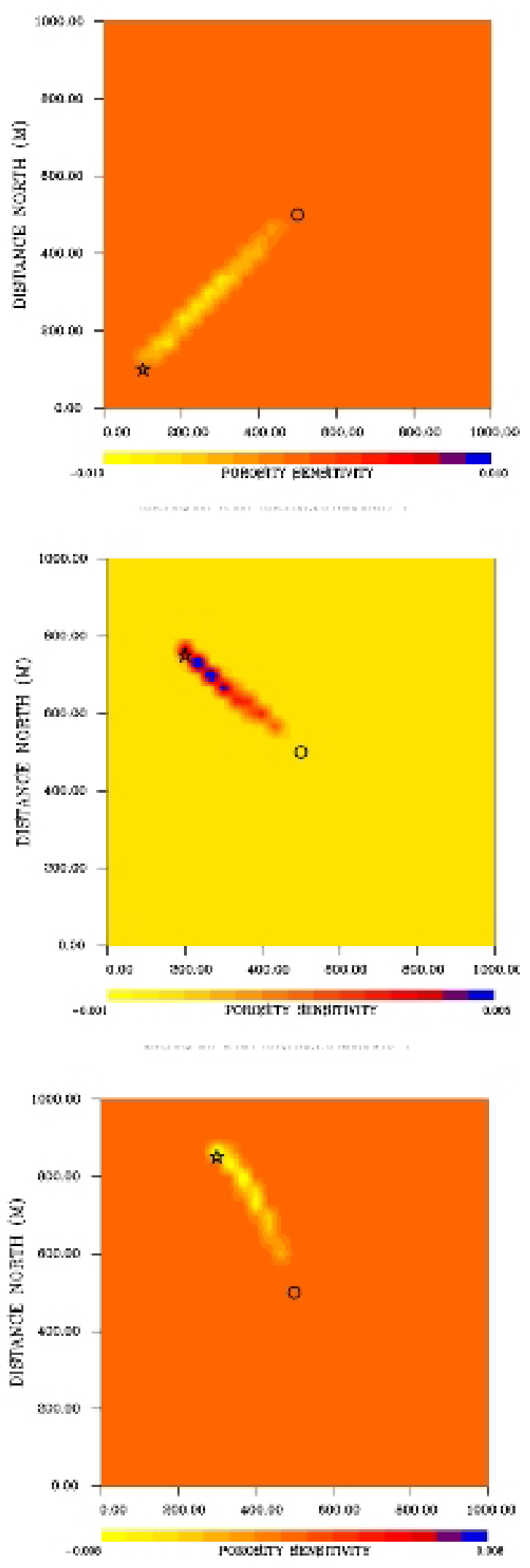


\section{Numeric}
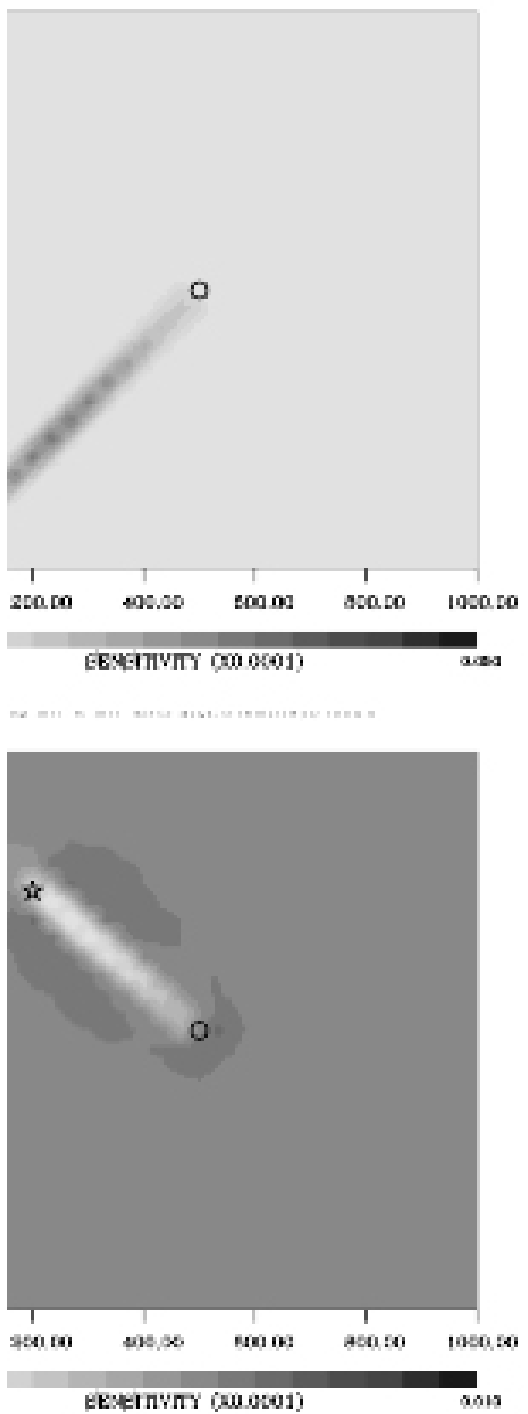

EExeInVIr (wotos)

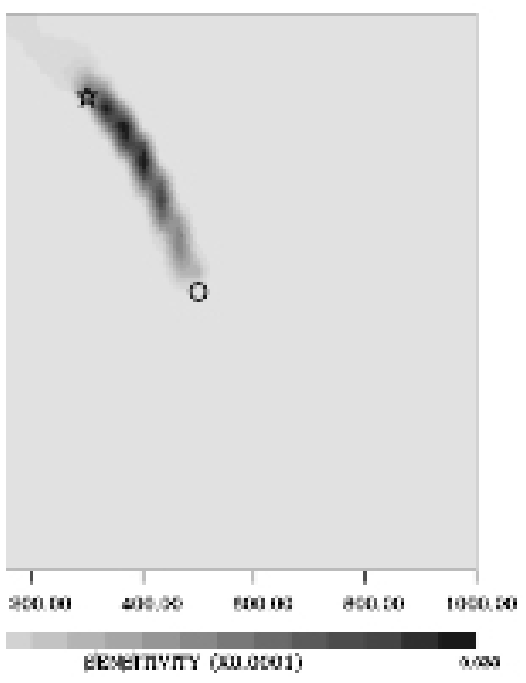

Analytic
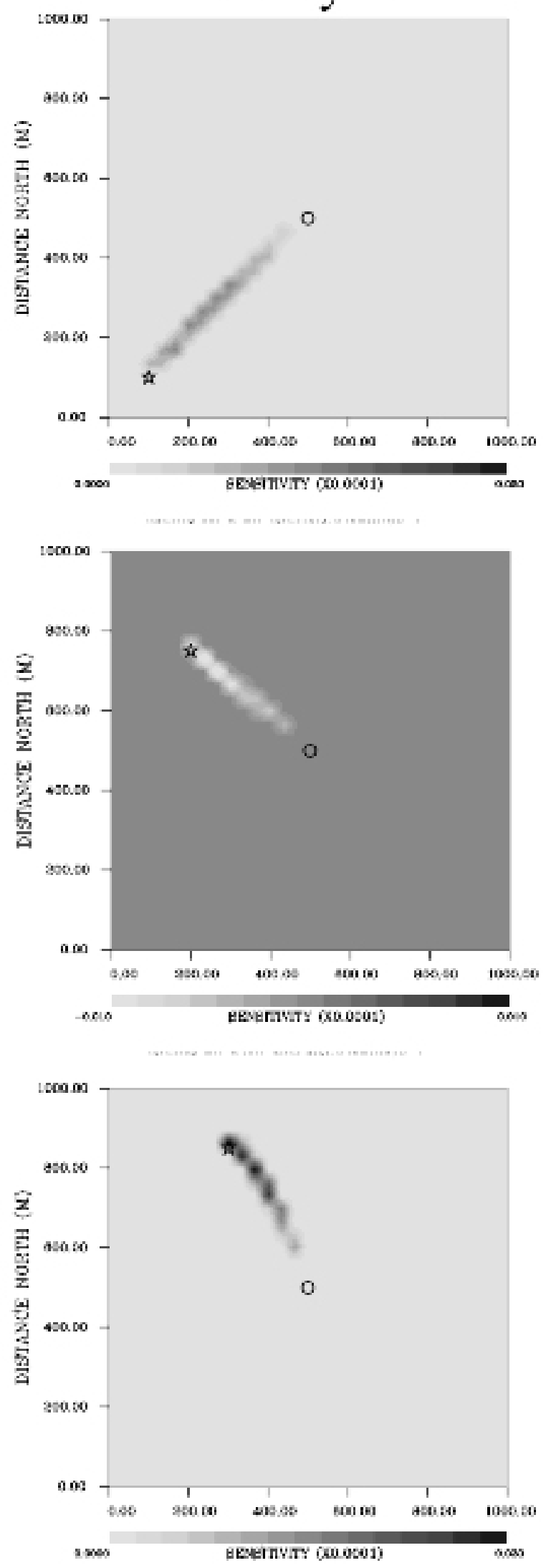


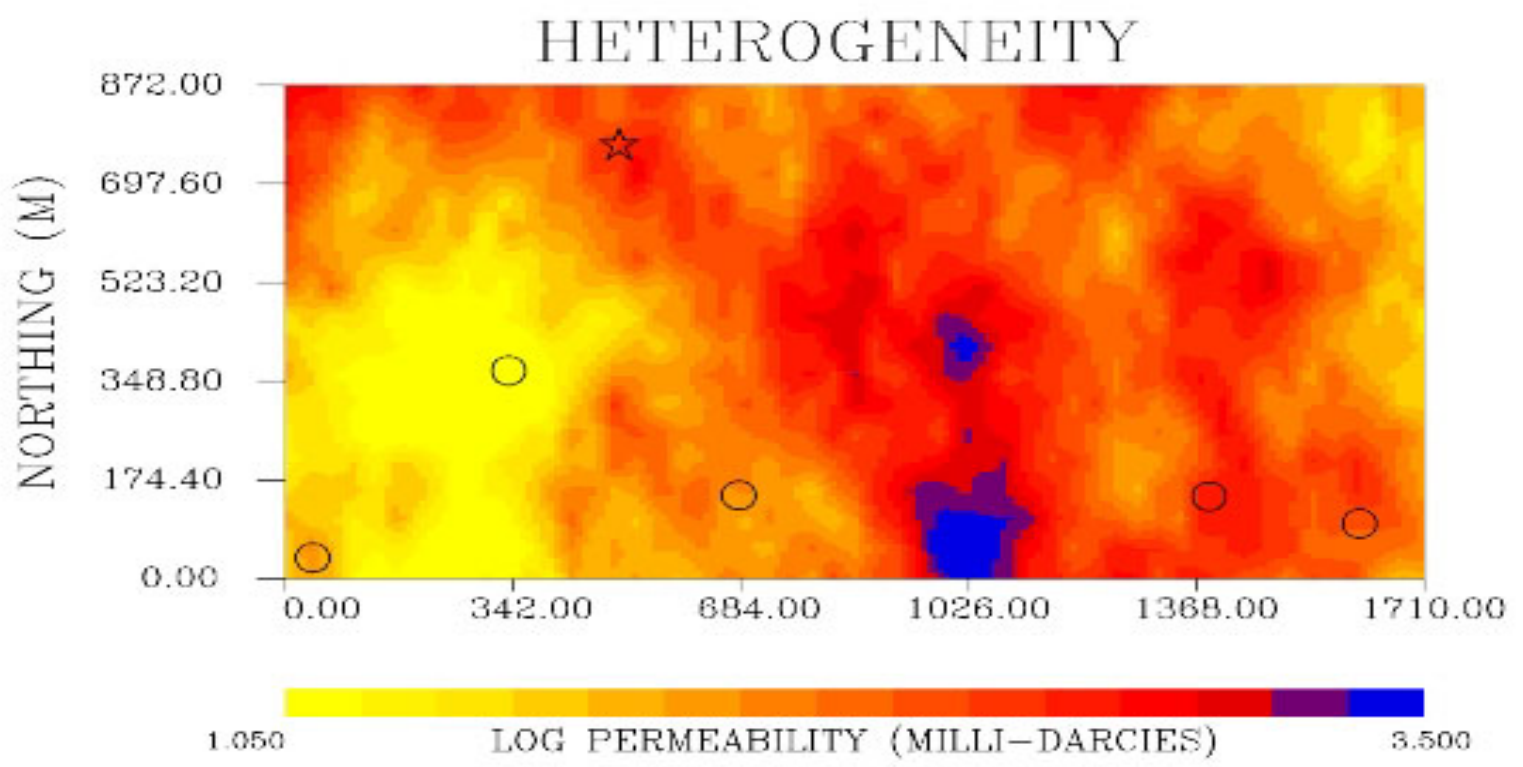



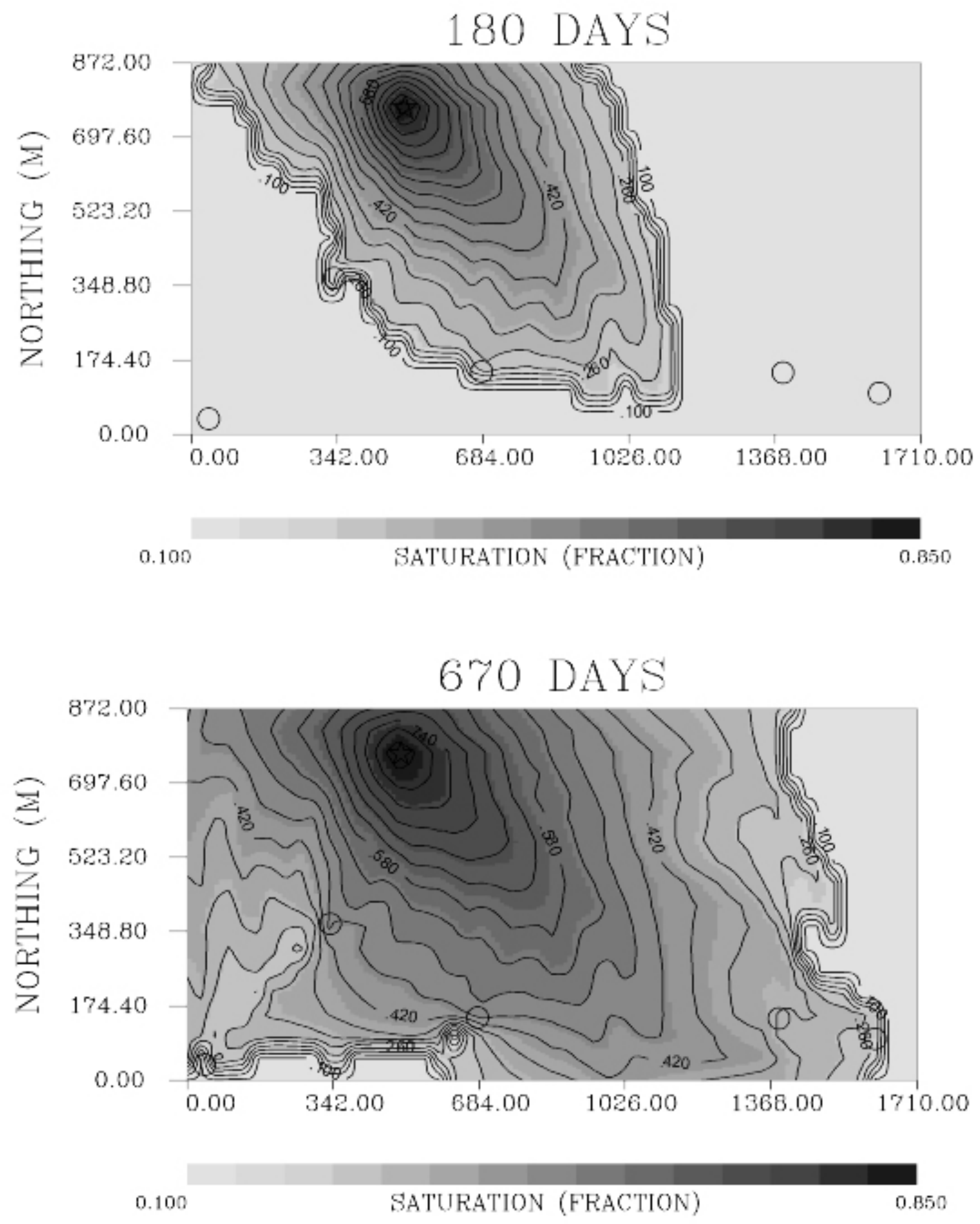


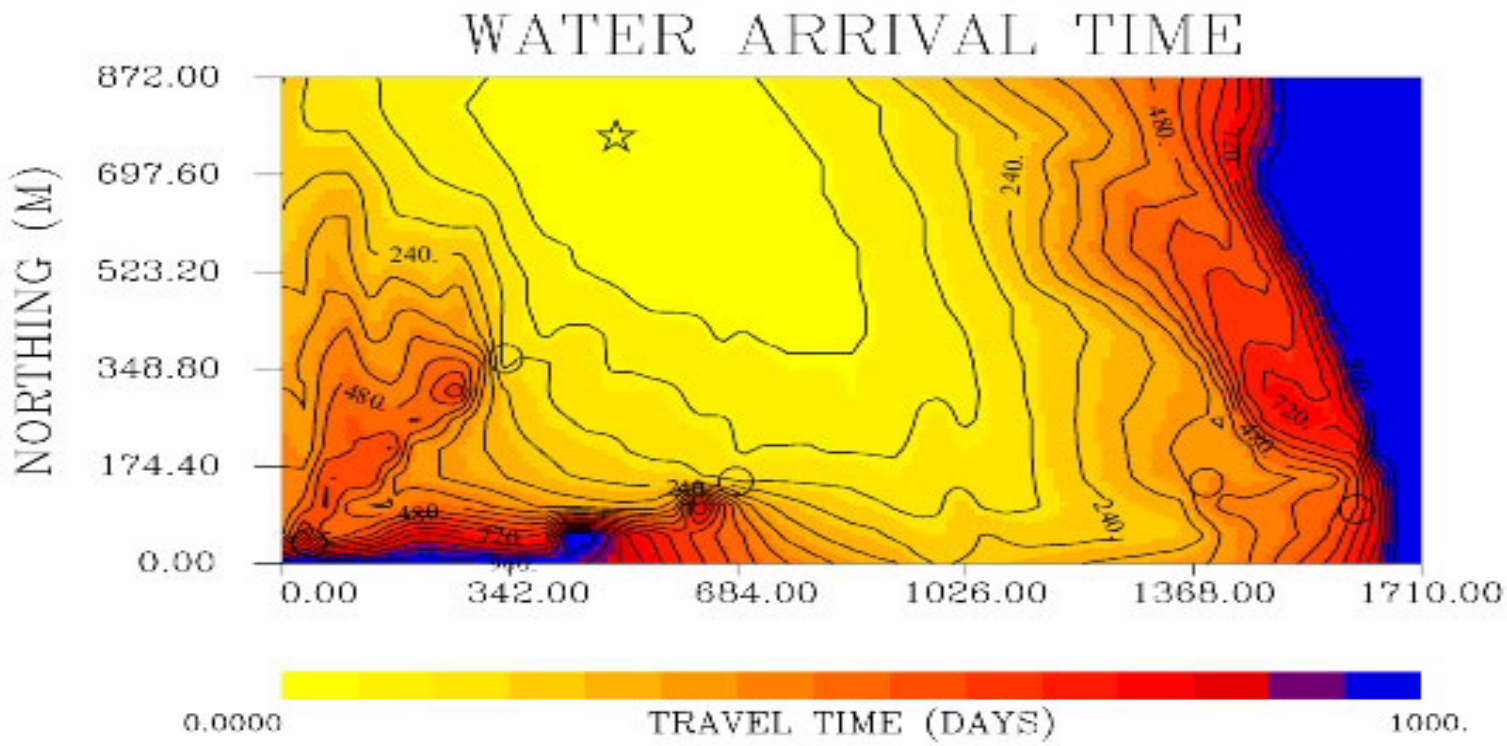




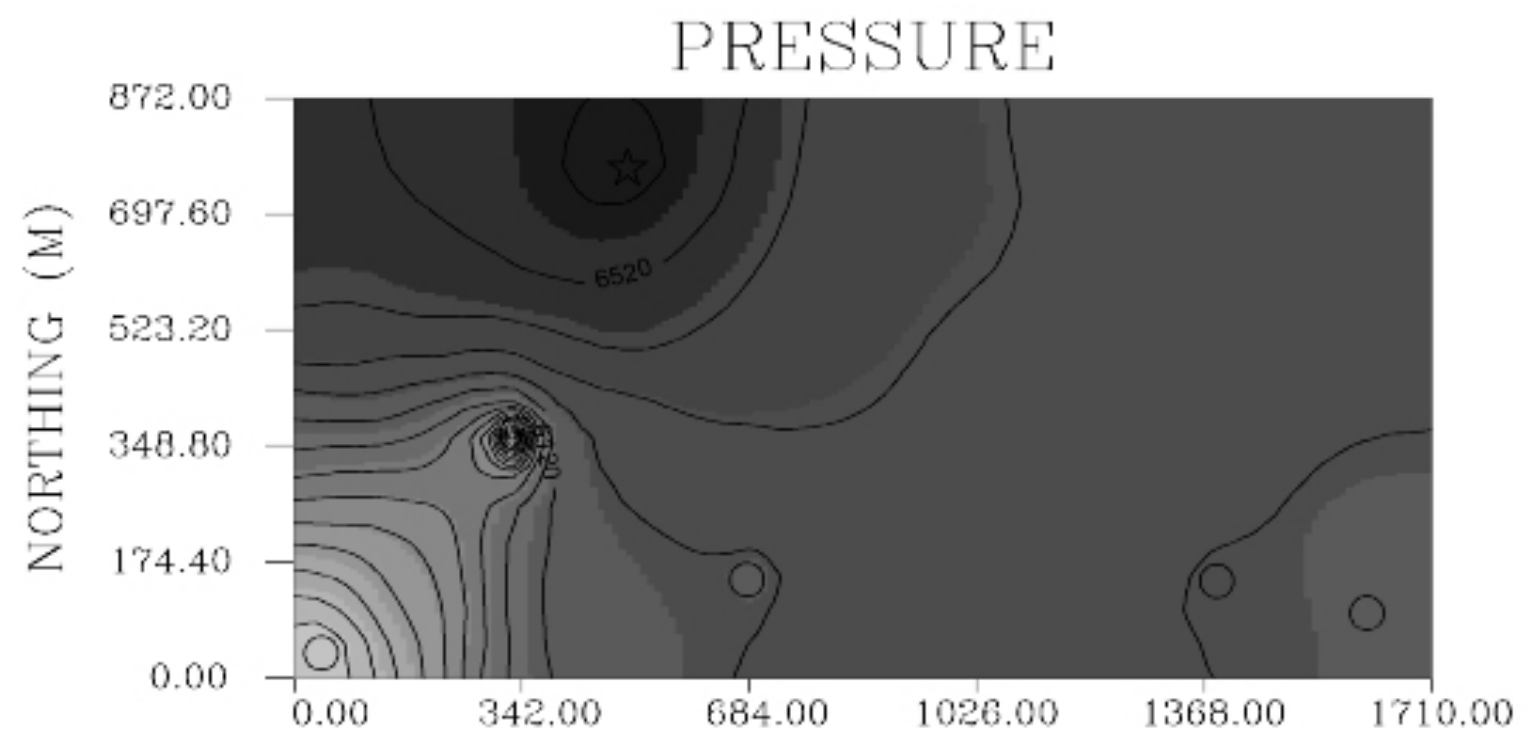

4820 .

PRESSURE (PSI)

6704

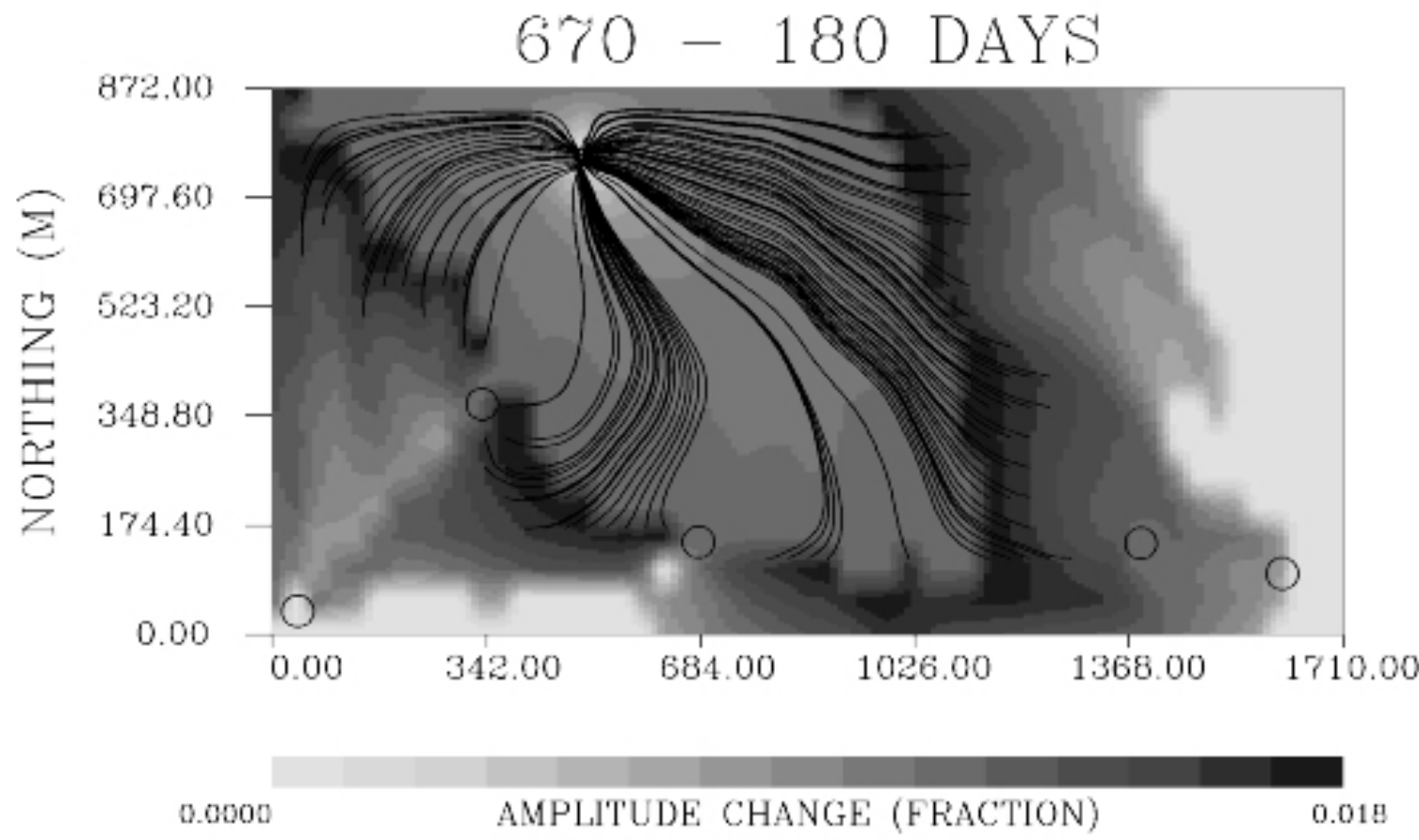




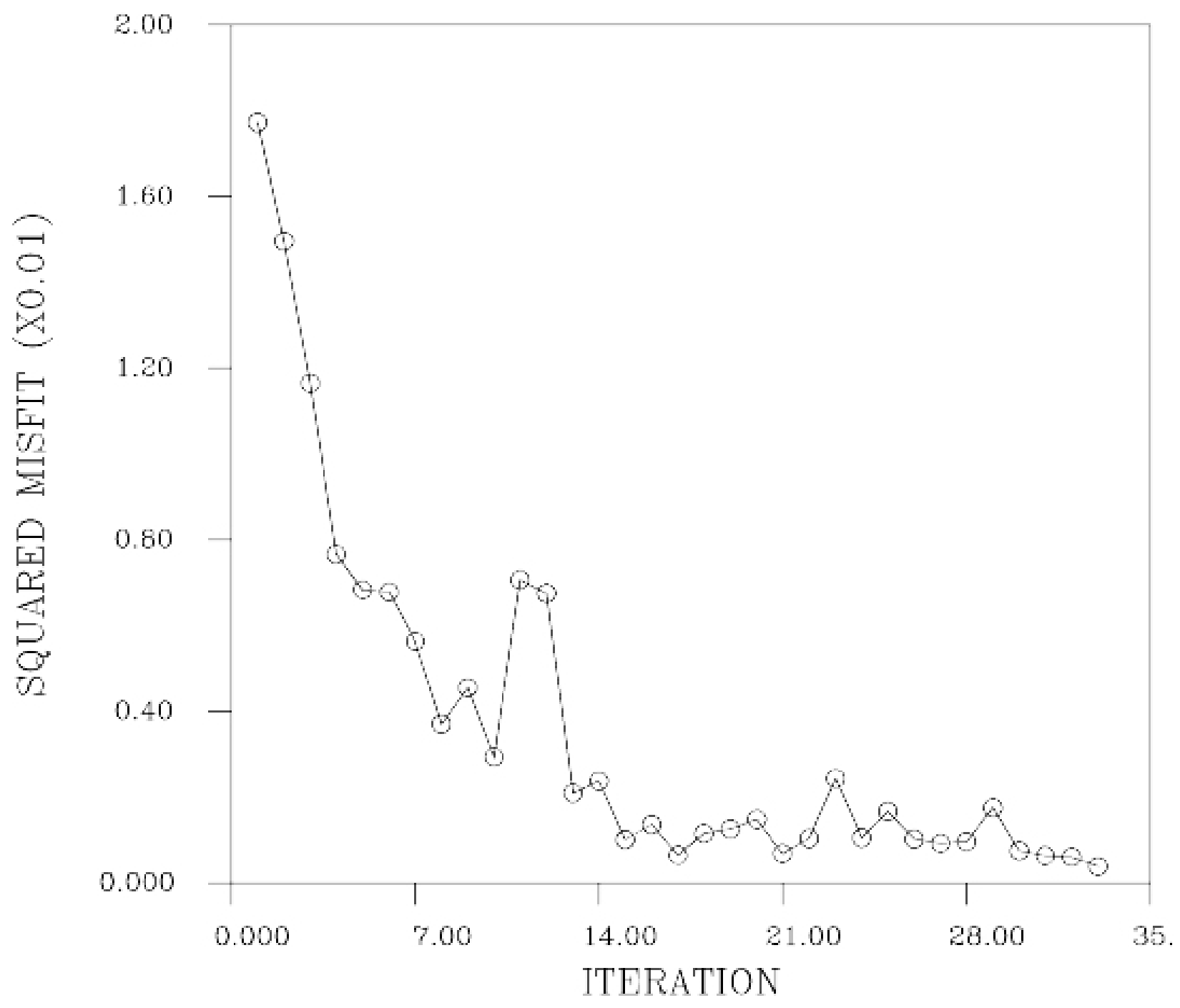




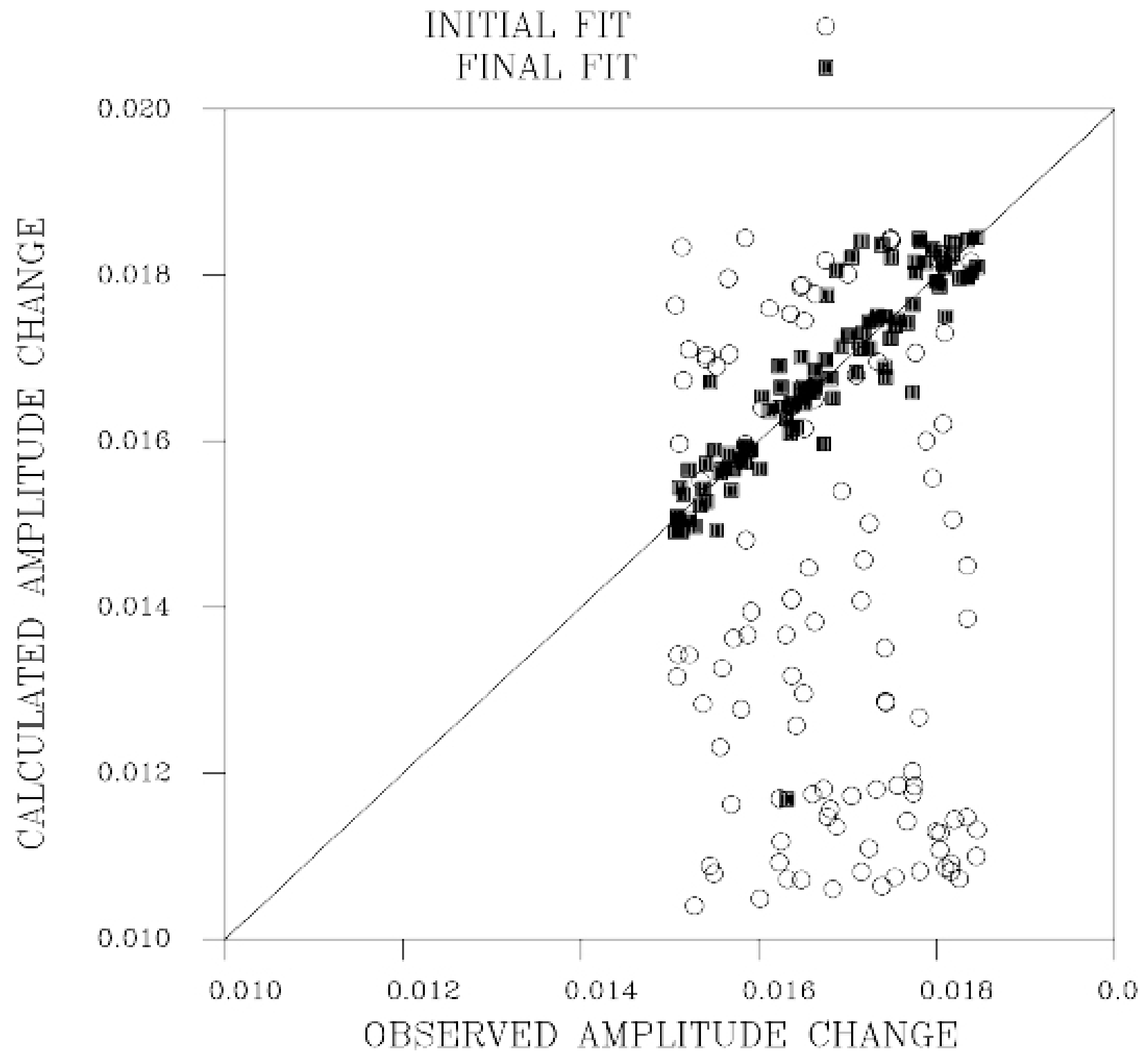




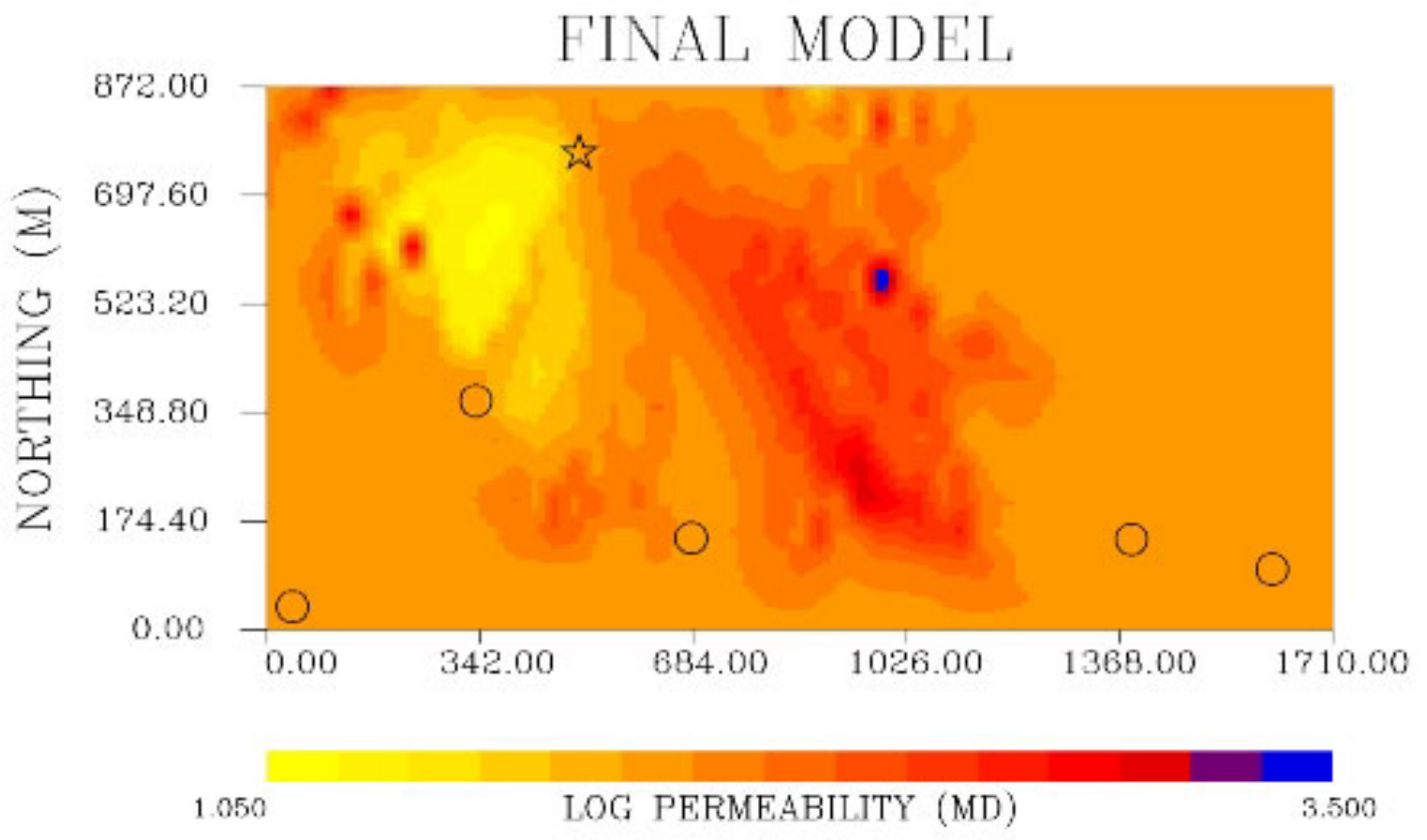




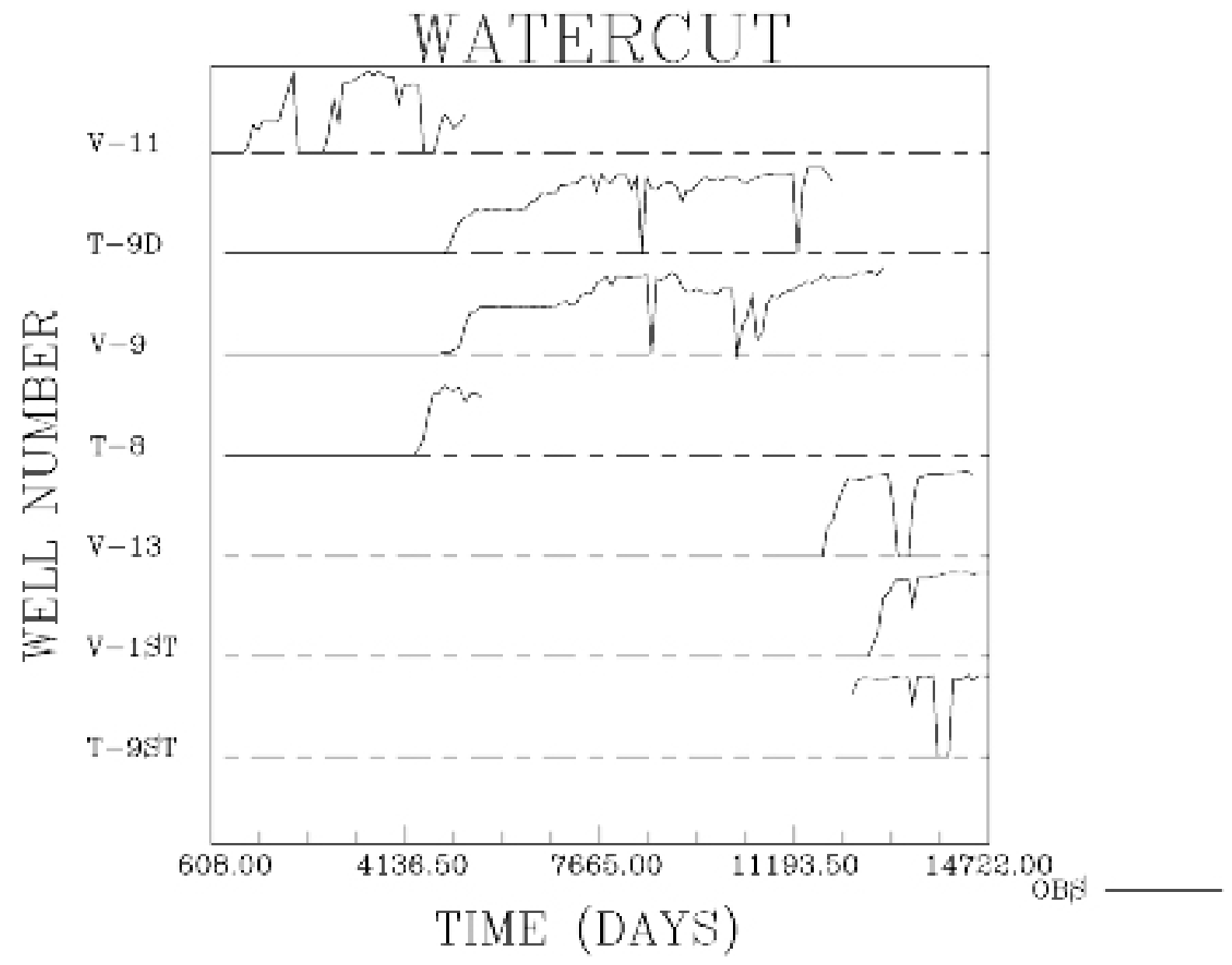




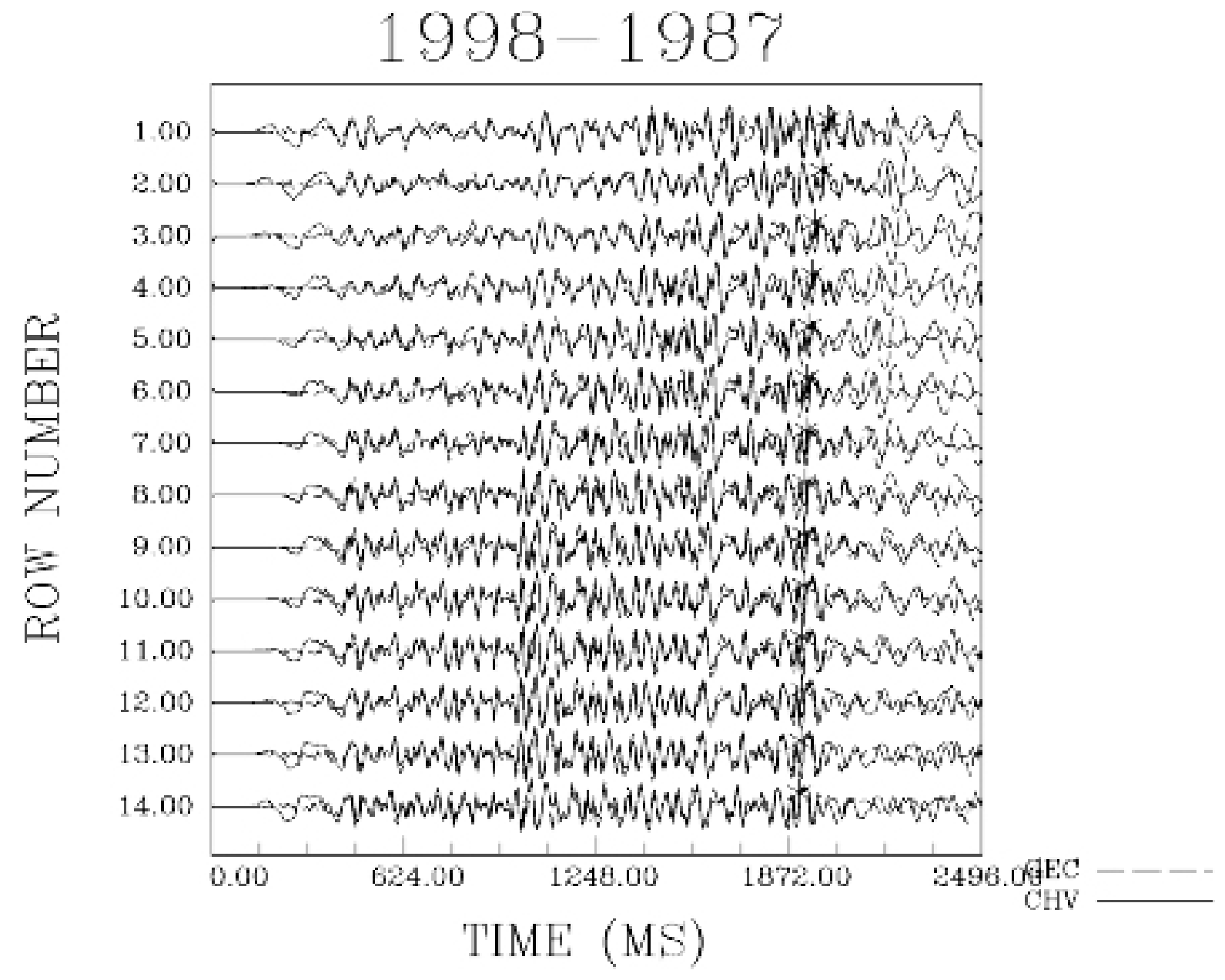




\section{OBSERVED AMPLITUDE CHANGE}
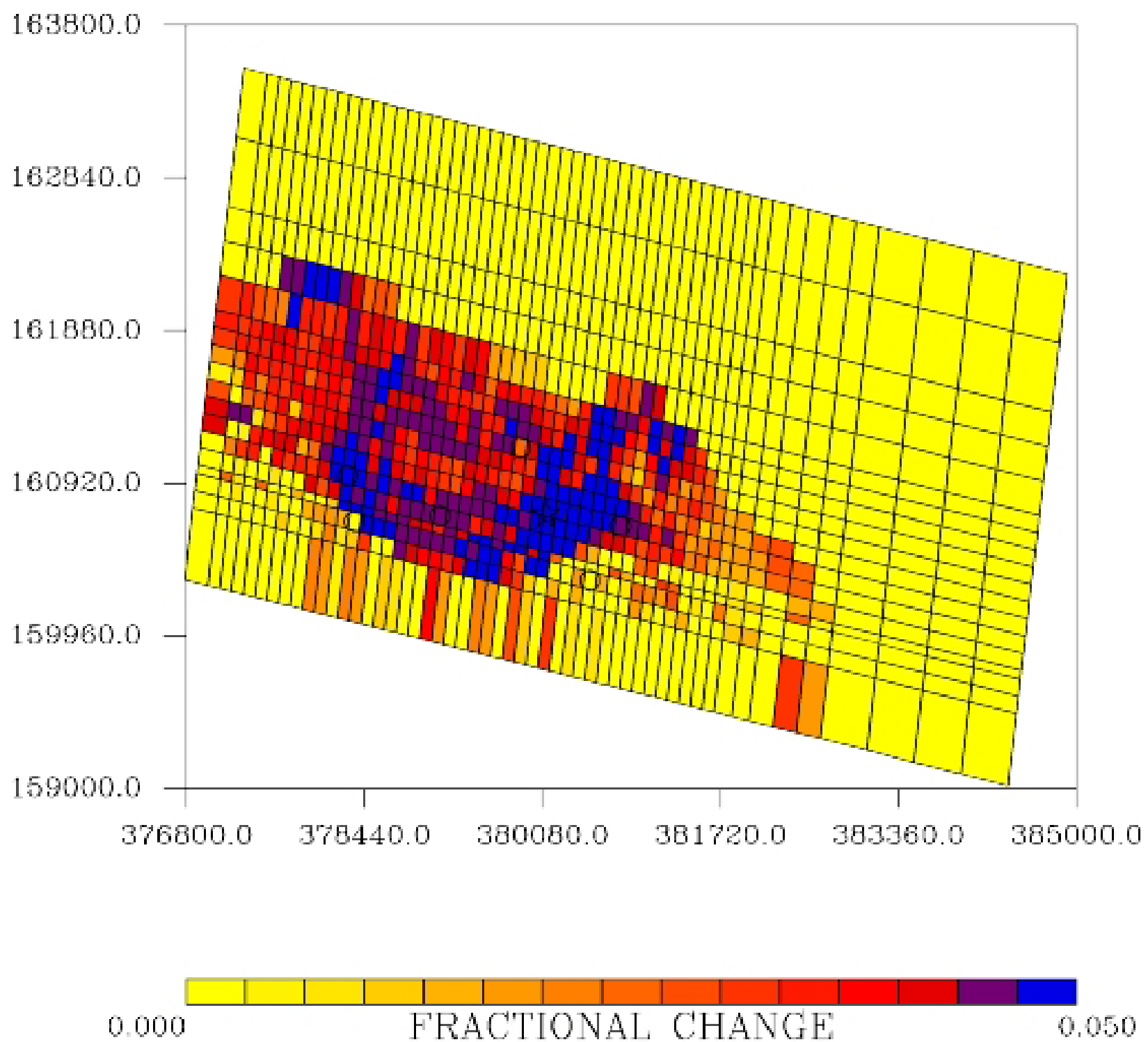


\section{INIT'IAL MUDEL}

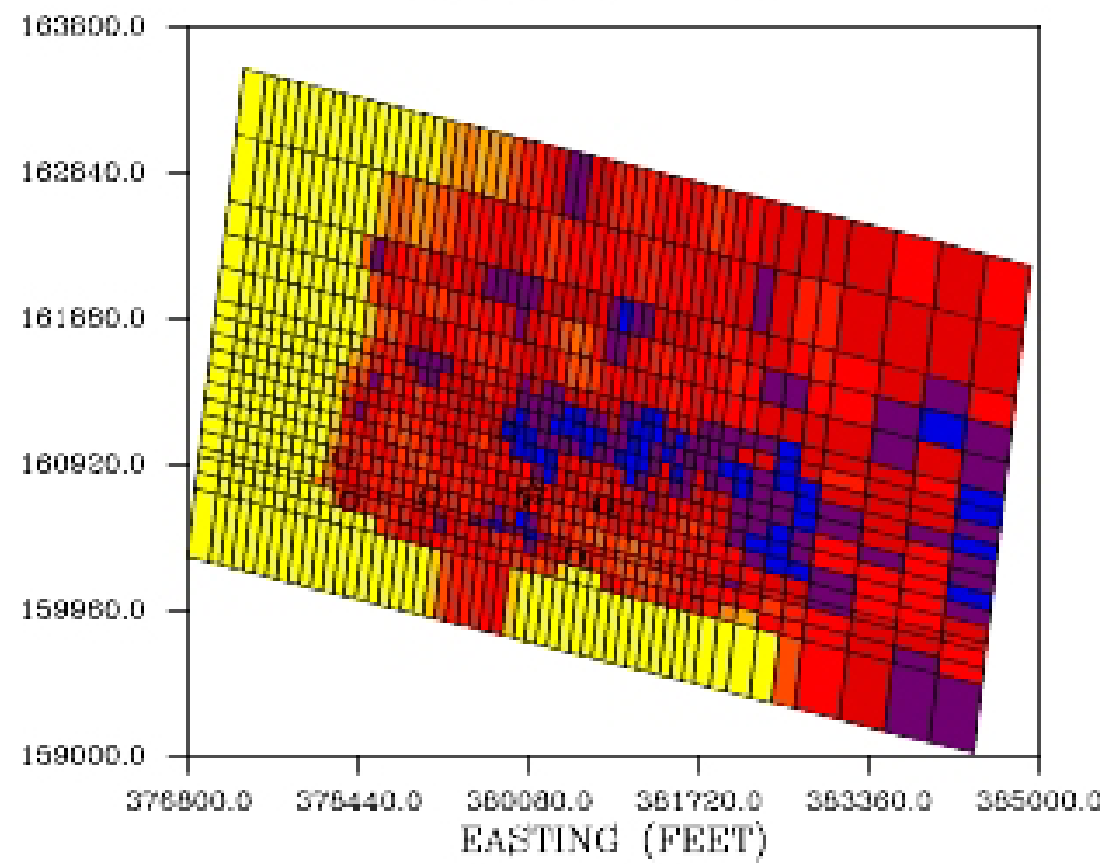

A.

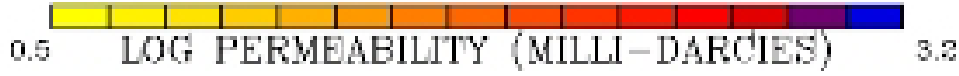

INITIAL WATER SATURATION

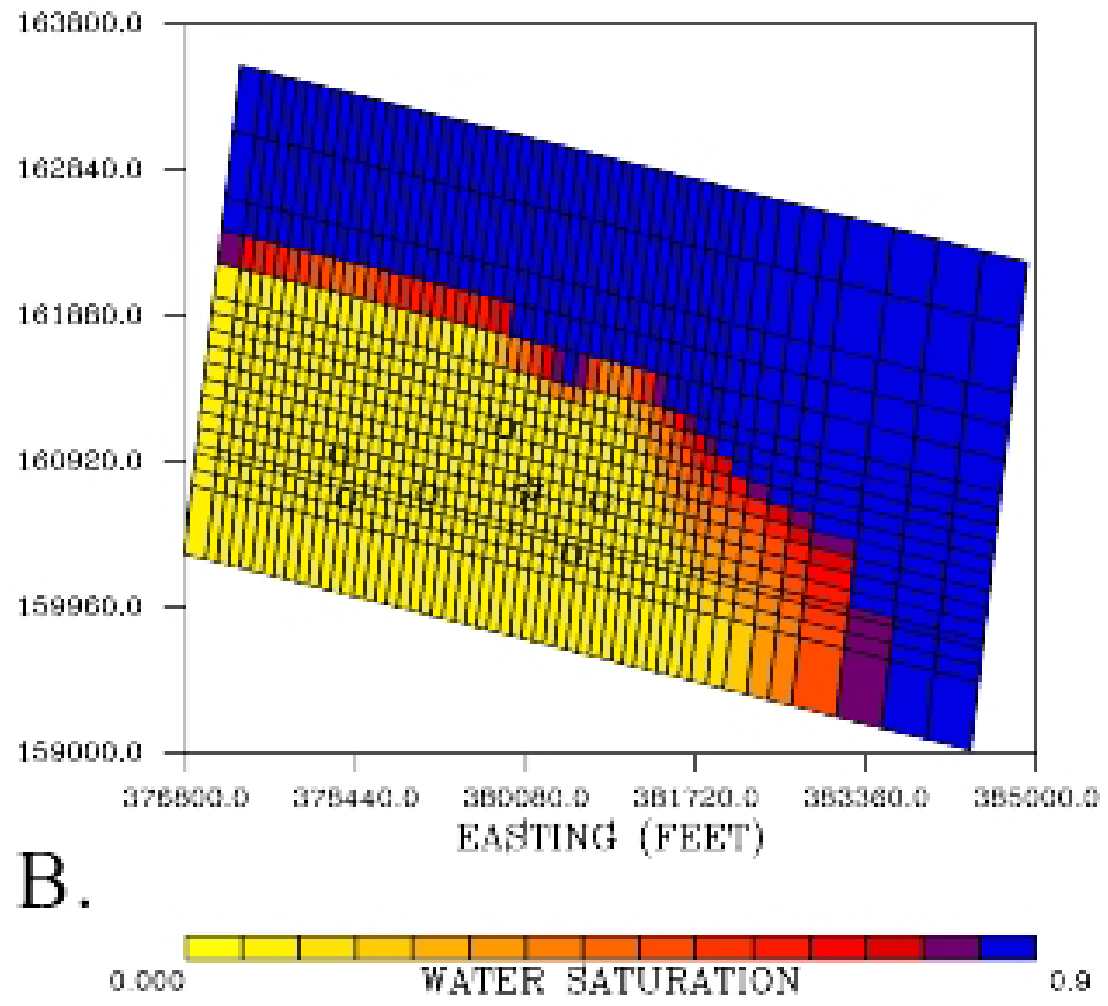




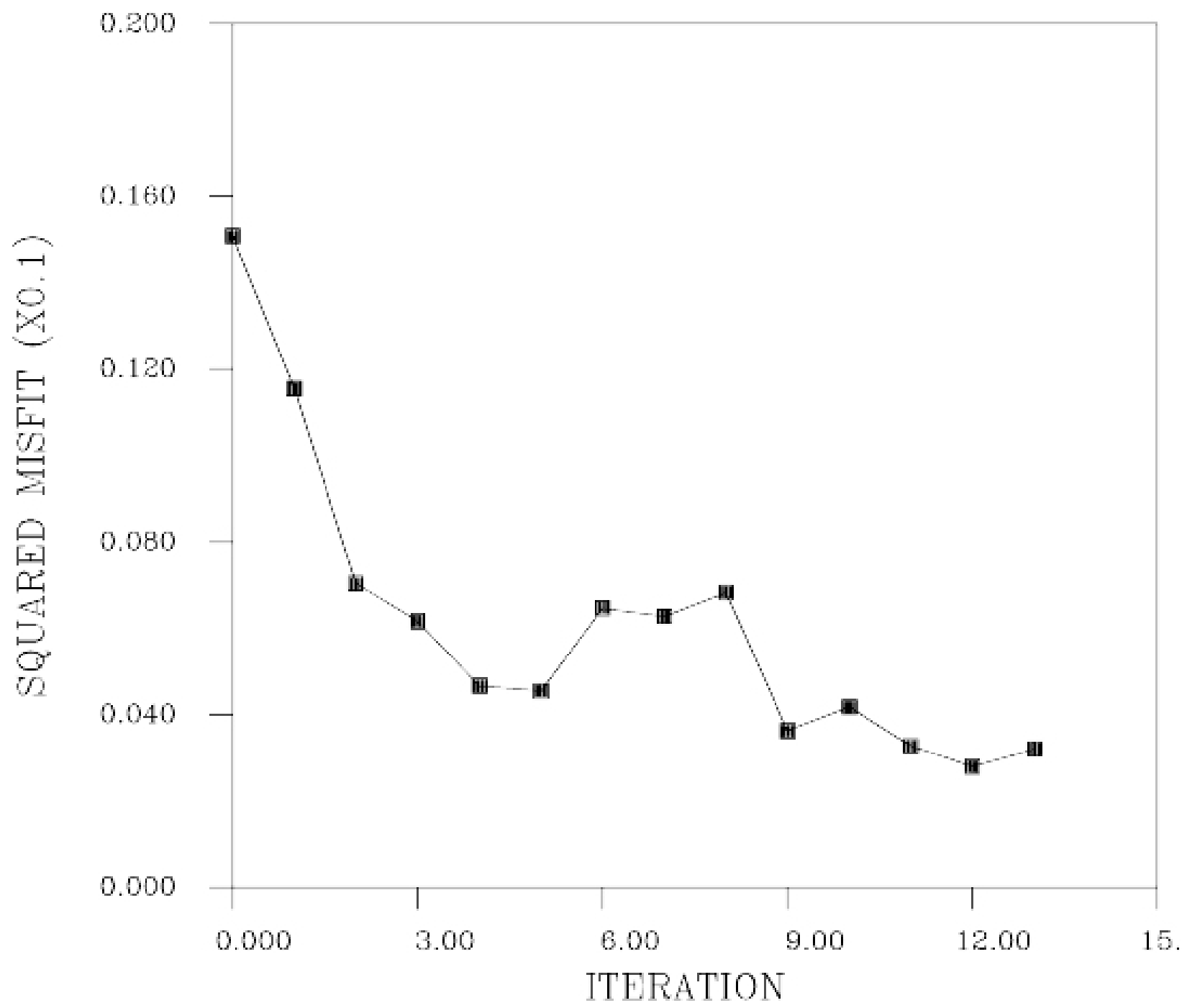




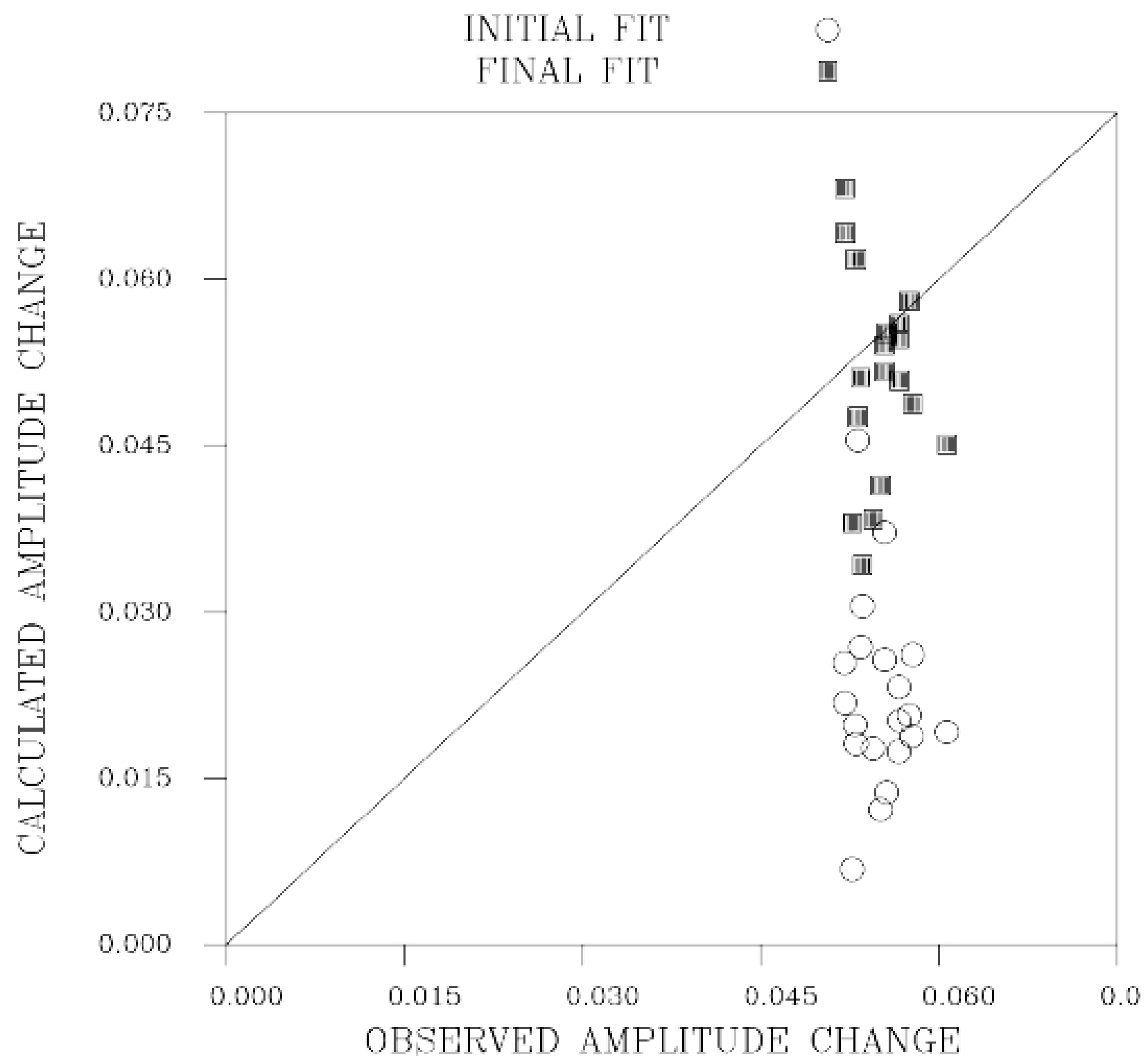


F'INAL MODELL
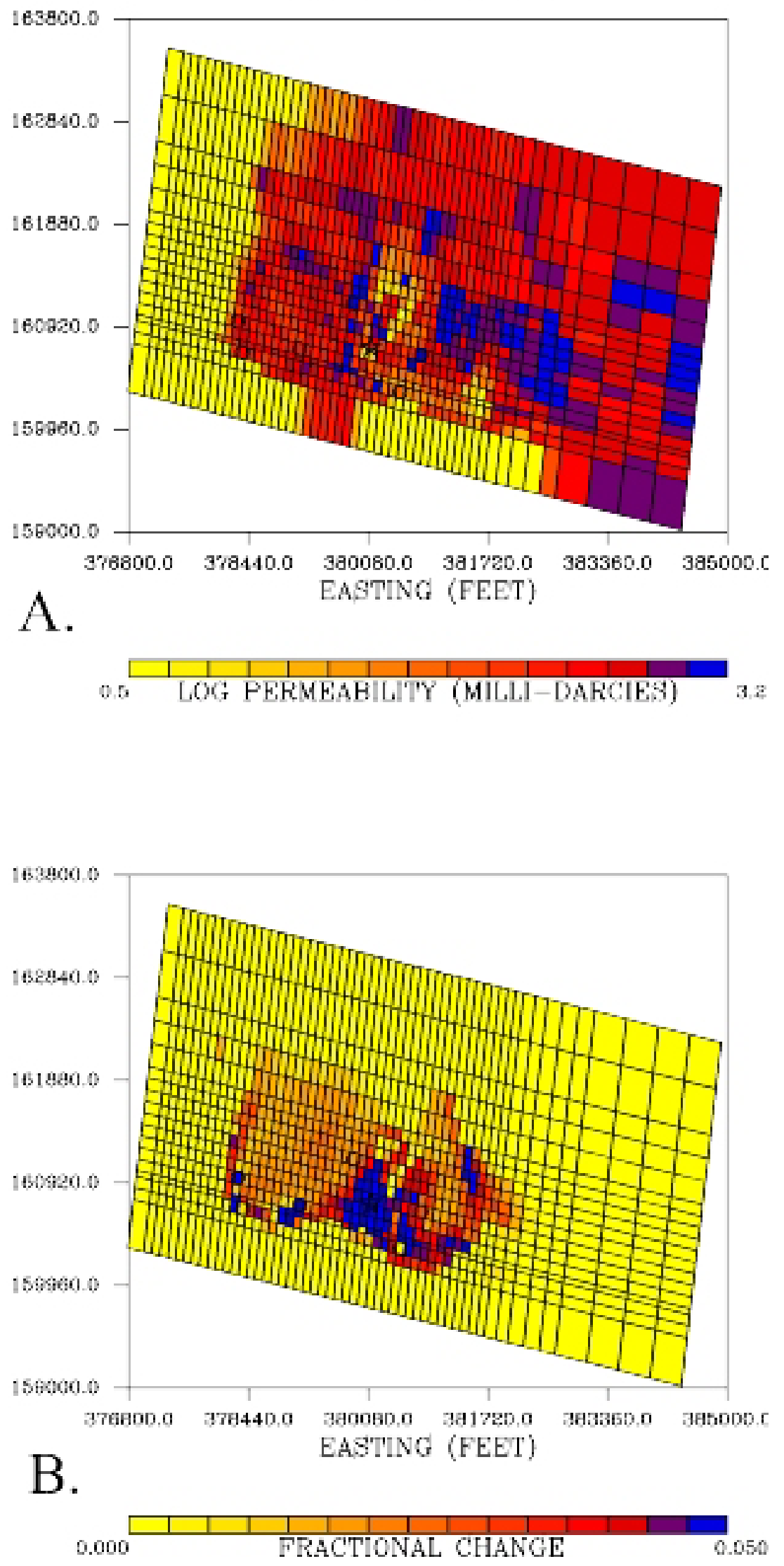